\title{
Undominated Groves Mechanisms
}

Mingyu Guo

University of Liverpool, UK

Evangelos Markakis

Athens University of Economics and Business, Greece

Krzysztof R. Apt

$C W I$, the Netherlands

Vincent Conitzer

Duke University, USA
Mingyu.GuO@LIVERPOOL.AC.UK

MARKAKIS@GMAIL.COM

APT@CWI.NL

CONITZER@CS.DUKE.EDU

\begin{abstract}
The family of Groves mechanisms, which includes the well-known VCG mechanism (also known as the Clarke mechanism), is a family of efficient and strategy-proof mechanisms. Unfortunately, the Groves mechanisms are generally not budget balanced. That is, under such mechanisms, payments may flow into or out of the system of the agents, resulting in deficits or reduced utilities for the agents. We consider the following problem: within the family of Groves mechanisms, we want to identify mechanisms that give the agents the highest utilities, under the constraint that these mechanisms must never incur deficits.
\end{abstract}

We adopt a prior-free approach. We introduce two general measures for comparing mechanisms in prior-free settings. We say that a non-deficit Groves mechanism $M$ individually dominates another non-deficit Groves mechanism $M^{\prime}$ if for every type profile, every agent's utility under $M$ is no less than that under $M^{\prime}$, and this holds with strict inequality for at least one type profile and one agent. We say that a non-deficit Groves mechanism $M$ collectively dominates another non-deficit Groves mechanism $M^{\prime}$ if for every type profile, the agents' total utility under $M$ is no less than that under $M^{\prime}$, and this holds with strict inequality for at least one type profile. The above definitions induce two partial orders on non-deficit Groves mechanisms. We study the maximal elements corresponding to these two partial orders, which we call the individually undominated mechanisms and the collectively undominated mechanisms, respectively.

\section{Introduction}

Mechanism design is often employed for coordinating group decision making among agents. Often, such mechanisms impose payments that agents have to pay to a central authority. Although maximizing revenue is a desirable objective in many settings (for example, if the mechanism is an auction designed by the seller), it is not desirable in situations where no entity is profiting from the payments. Some examples include public project problems as well as certain resource allocation problems without a seller (e.g., the right to use a shared good in a given time slot, or the assignment of take-off slots among airline companies). In such cases, we would like to have mechanisms that minimize payments (or, even better, achieve budget balance), while maintaining other desirable properties, such as being efficient, strategy-proof and non-deficit (i.e., the mechanism does not need to be funded by an external source). 
The family of Groves mechanisms, which includes the well-known VCG mechanism (also known as the Clarke mechanism), is a family of efficient and strategy-proof mechanisms. In many sufficiently general settings, including the settings that we will study in this paper, the Groves mechanisms are the only efficient and strategy-proof mechanisms (Holmström, 1979). Unfortunately though, the Groves mechanisms are generally not budget balanced. That is, under such mechanisms, payments may flow into or out of the system of the agents, resulting in deficits or reduced utilities for the agents. Motivated by this we consider in this paper the following problem: within the family of Groves mechanisms, we want to identify mechanisms that give the agents the highest utilities, under the constraint that these mechanisms never incur deficits. ${ }^{1}$

We adopt a prior-free approach, where each agent $i$ knows only his own valuation $v_{i}$, and there is no prior probability distribution over the other agents' values. We introduce two natural measures for comparing mechanisms in prior-free settings. Given a performance indicator, we say that mechanism $M$ individually dominates mechanism $M^{\prime}$ if for every type profile of the agents, $M$ performs no worse than $M^{\prime}$ from the perspective of each individual agent, and this holds with strict inequality for at least one type profile and one agent. We say that mechanism $M$ collectively dominates mechanism $M^{\prime}$ if for every type profile, $M$ performs no worse than $M^{\prime}$ from the perspective of the set of agents as a whole, and this holds with strict inequality for at least one type profile. In this paper, we focus on maximizing the agents' utilities. Given this specific performance indicator, individual and collective dominance are determined by comparing either individual utilities or the sum of the agents' utilities, respectively.

The above definitions induce two partial orders on non-deficit Groves mechanisms. Our goal in this work is to identify and study the maximal elements corresponding to these two partial orders, which we call the individually undominated (non-deficit Groves) mechanisms and the collectively undominated (non-deficit Groves) mechanisms, respectively. It should be noted that the partial orders we focus on may be different from the partial orders induced by other performance indicators, e.g., if the criterion is the revenue extracted from the agents.

\subsection{Structure of the Paper}

The presentation of our results is structured as follows: In Sections 2 and 3, we formally define the notions of individual and collective dominance, as well as the family of Groves mechanisms, and then provide some basic observations. We also establish some general properties of anonymous Groves mechanisms which we use later on, and which may be of independent interest. We then begin our study of individual dominance in Section 4, where we give a characterization of individually undominated mechanisms. We also propose two techniques for transforming any given non-deficit Groves mechanism into one that is individually undominated.

In Sections 5 and 6 we study the question of finding collectively undominated mechanisms in two settings. The first (Section 5) is auctions of multiple identical units with unit-demand bidders. In this setting, the VCG mechanism is collectively dominated by

1. The agents' utilities may be further increased if we also consider mechanisms outside of the Groves family (Guo \& Conitzer, 2008a; de Clippel, Naroditskiy, \& Greenwald, 2009; Faltings, 2005; Guo, Naroditskiy, Conitzer, Greenwald, \& Jennings, 2011), but in this paper we take efficiency as a hard constraint. 
other non-deficit Groves mechanisms, such as the Bailey-Cavallo mechanism (Bailey, 1997; Cavallo, 2006). We obtain a complete characterization of collectively undominated mechanisms that are anonymous and linear (meaning that the redistribution is a linear function of the ordered type profile; see Section 5 for the definition). In particular, we show that the collectively undominated mechanisms that are anonymous and linear are exactly the Optimal-in-Expectation Linear (OEL) redistribution mechanisms, which include the BaileyCavallo mechanism and were introduced by Guo and Conitzer (2010). The second setting (Section 6) is public project problems, where agents must decide on whether and how to finance a project. We show that in the case where the agents have identical participation costs, the VCG mechanism is collectively undominated. On the other hand, when the participation costs can be different across agents, there exist mechanisms that collectively dominate VCG. We finally show that when the participation costs are different across agents, the VCG mechanism remains collectively undominated among all pay-only mechanisms.

\subsection{Related Work}

How to efficiently allocate resources among a group of competing agents is a well-studied topic in economics literature. For example, the famous Myerson-Satterthwaite Theorem (Myerson \& Satterthwaite, 1983) rules out the existence of efficient, Bayes-Nash incentive compatible, budget-balanced, and individually rational mechanisms. Cramton, Gibbons, and Klemperer (1987) characterized the Bayes-Nash incentive compatible and individually rational mechanisms for dissolving a partnership, and gave the necessary and sufficient condition for the possibility of dissolving partnership efficiently.

The main difference between these papers and ours is that we adopt a prior-free approach. That is, we do not assume that we know the prior distribution of the agents' valuations. As a result of this, our notion of truthfulness is strategy-proofness, which is stronger than Bayes-Nash incentive compatibility. In many sufficiently general settings, including the settings that we will study in this paper, the Groves mechanisms are the only efficient and strategy-proof mechanisms (Holmström, 1979). That is, the search of undominated Groves mechanisms is, in many settings, the search of efficient, strategy-proof, and non-deficit mechanisms that are closest to budget-balance.

Recently, there has been a series of works on VCG redistribution mechanisms, which are mechanisms that make social decisions according to the efficient and strategy-proof VCG mechanism, and then redistribute some of the VCG payments back to the agents, under certain constraints, such as that an agent's redistribution should be independent of his own type (therefore ensuring strategy-proofness), and that the total redistribution should never exceed the total VCG payment (therefore ensuring non-deficit). Actually, any non-deficit Groves mechanism can be interpreted as such a VCG-based redistribution mechanism, and any (non-deficit) VCG redistribution mechanism corresponds to a nondeficit Groves mechanism (more details on this are provided in Section 2).

One example of a redistribution mechanism is the Bailey-Cavallo (BC) mechanism (Cavallo, 2006). ${ }^{2}$ Under the BC mechanism, every agent, besides participating in the VCG

2. In settings that are revenue monotonic, the Cavallo (2006) mechanism coincides with a mechanism discovered earlier by Bailey (1997). The Bailey-Cavallo mechanism for a single-item auction was also independently discovered by Porter, Shoham, and Tennenholtz (2004). 
mechanism, also receives $\frac{1}{n}$ times the minimal VCG revenue that could have been obtained by changing this agent's own bid. In some settings (e.g., a single-item auction), the BC mechanism can successfully redistribute a large portion of the VCG payments back to the agents. That is, in such settings, the BC mechanism both individually and collectively dominates the VCG mechanism.

Guo and Conitzer (2009) proposed another VCG redistribution mechanism called the worst-case optimal (WCO) redistribution mechanism, in the setting of multi-unit auctions with nonincreasing marginal values. WCO is optimal in terms of the fraction of total VCG payment redistributed in the worst case. ${ }^{3}$ Moulin (2009) independently derived WCO under a slightly different worst-case optimality notion (in the more restrictive setting of multi-unit auctions with unit demand only). Guo and Conitzer (2010) also proposed a family of VCG redistribution mechanisms that aim to maximize the expected amount of VCG payment redistributed, in the setting of multi-unit auctions with unit demand. The members of this family are called the Optimal-in-Expectation Linear (OEL) redistribution mechanisms.

Finally, the paper that is the closest to what we study here is an early work by Moulin on collectively undominated non-deficit Groves mechanisms (Moulin, 1986). It deals with the problem of selecting an efficient public decision out of finitely many costless alternatives. ${ }^{4}$ Each agent submits to the central authority his utility for each alternative. Subsequently, the central authority makes a decision that maximizes the social welfare. Moulin (1986, Lemma 2) showed that the VCG mechanism is collectively undominated in the above setting. This result generalizes an earlier result for the case of two public decisions by Laffont and Maskin (1997).

\section{Preliminaries}

We first briefly review payment-based mechanisms (see, e.g., Mas-Colell, Whinston, \& Green, 1995).

\subsection{Payment-Based Mechanisms}

Assume that there is a set of possible outcomes or decisions $D$, a set $\{1, \ldots, n\}$ of agents where $n \geq 2$, and for each agent $i$, a set of types $\Theta_{i}$ and an (initial) utility function $v_{i}: D \times \Theta_{i} \rightarrow \mathbb{R}$. Let $\Theta:=\Theta_{1} \times \cdots \times \Theta_{n}$.

In a (direct revelation) mechanism, each agent reports a type $\theta_{i} \in \Theta_{i}$ and based on this, the mechanism selects an outcome and a payment to be made by every agent. Hence a mechanism is given by a pair of functions $(f, t)$, where $f$ is the decision function and $t$ is the payment function that determines the agents' payments, i.e., $f: \Theta \rightarrow D$, and $t: \Theta \rightarrow \mathbb{R}^{n}$.

We put $t_{i}(\theta):=(t(\theta))_{i}$, i.e., the function $t_{i}$ computes the payment of agent $i$. For each vector $\theta$ of announced types, if $t_{i}(\theta) \geq 0$, agent $i$ pays $t_{i}(\theta)$, and if $t_{i}(\theta)<0$, he receives $\left|t_{i}(\theta)\right|$. When the true type of agent $i$ is $\theta_{i}$ and his announced type is $\theta_{i}^{\prime}$, his final utility function is defined by

$$
u_{i}\left((f, t)\left(\theta_{i}^{\prime}, \theta_{-i}\right), \theta_{i}\right):=v_{i}\left(f\left(\theta_{i}^{\prime}, \theta_{-i}\right), \theta_{i}\right)-t_{i}\left(\theta_{i}^{\prime}, \theta_{-i}\right),
$$

3. This notion of worst-case optimality was also studied for more general settings (Gujar \& Narahari, 2011; Guo, 2011, 2012).

4. In our public project model, there is a cost associated with building the project. 
where $\theta_{-i}$ is the vector of types announced by the other agents.

\subsection{Properties of Payment-Based Mechanisms}

We say that a payment-based mechanism $(f, t)$ is

- efficient if for all $\theta \in \Theta$ and $d \in D, \sum_{i=1}^{n} v_{i}\left(f(\theta), \theta_{i}\right) \geq \sum_{i=1}^{n} v_{i}\left(d, \theta_{i}\right)$,

- budget-balanced if $\sum_{i=1}^{n} t_{i}(\theta)=0$ for all $\theta \in \Theta$,

- non-deficit if $\sum_{i=1}^{n} t_{i}(\theta) \geq 0$ for all $\theta$, i.e., the mechanism does not need to be funded by an external source,

- pay-only if $t_{i}(\theta) \geq 0$ for all $\theta$ and all $i \in\{1, \ldots, n\}$,

- strategy-proof if for all $\theta, i \in\{1, \ldots, n\}$, and $\theta_{i}^{\prime}$,

$$
u_{i}\left((f, t)\left(\theta_{i}, \theta_{-i}\right), \theta_{i}\right) \geq u_{i}\left((f, t)\left(\theta_{i}^{\prime}, \theta_{-i}\right), \theta_{i}\right),
$$

i.e., for each agent $i$, reporting a false type, here $\theta_{i}^{\prime}$, is not profitable.

\subsection{Individual and Collective Dominance}

We consider prior-free settings, where each agent $i$ knows only his own function $v_{i}$, and there is no belief or prior probability distribution regarding the other agents' initial utilities. Payment-based mechanisms can naturally be compared in terms of either the effect on each individual agent or the global effect on the whole set of agents. We therefore introduce two measures for comparing such mechanisms. Given a performance indicator ${ }^{5}$, we say that mechanism $\left(f^{\prime}, t^{\prime}\right)$ individually dominates mechanism $(f, t)$ if for every type profile, $\left(f^{\prime}, t^{\prime}\right)$ performs no worse than $(f, t)$ from the perspective of every agent, and this holds with strict inequality for at least one type profile and one agent. We say that mechanism $\left(f^{\prime}, t^{\prime}\right)$ collectively dominates mechanism $(f, t)$ if for every type profile, $\left(f^{\prime}, t^{\prime}\right)$ performs no worse than $(f, t)$ from the perspective of the whole agent system, and this holds with strict inequality for at least one type profile. In this paper, we focus on maximizing the agents' utilities. Given this specific performance indicator, individual and collective dominance are captured by the following definitions:

Definition 2.1 Given two payment-based mechanisms $(f, t)$ and $\left(f^{\prime}, t^{\prime}\right)$, we say that $\left(f^{\prime}, t^{\prime}\right)$ individually dominates $(f, t)$ if

- for all $\theta \in \Theta$ and all $i \in\{1, \ldots, n\}, u_{i}\left((f, t)(\theta), \theta_{i}\right) \leq u_{i}\left(\left(f^{\prime}, t^{\prime}\right)(\theta), \theta_{i}\right)$,

- for some $\theta \in \Theta$ and some $i \in\{1, \ldots, n\}, u_{i}\left((f, t)(\theta), \theta_{i}\right)<u_{i}\left(\left(f^{\prime}, t^{\prime}\right)(\theta), \theta_{i}\right)$.

Definition 2.2 Given two payment-based mechanisms $(f, t)$ and $\left(f^{\prime}, t^{\prime}\right)$, we say that $\left(f^{\prime}, t^{\prime}\right)$ collectively dominates $(f, t)$ if

5. By a performance indicator we mean a function of the mechanism's outcome that serves as a measure for comparing mechanisms. E.g., it can be the final utility of an agent, or an arbitrary function of it, or a function of the agent's payment or any other function that depends on the decision rule and the payment rule of the mechanism. 
- for all $\theta \in \Theta, \sum_{i=1}^{n} u_{i}\left((f, t)(\theta), \theta_{i}\right) \leq \sum_{i=1}^{n} u_{i}\left(\left(f^{\prime}, t^{\prime}\right)(\theta), \theta_{i}\right)$,

- for some $\theta \in \Theta, \sum_{i=1}^{n} u_{i}\left((f, t)(\theta), \theta_{i}\right)<\sum_{i=1}^{n} u_{i}\left(\left(f^{\prime}, t^{\prime}\right)(\theta), \theta_{i}\right)$.

For two payment-based mechanisms $(f, t)$ and $\left(f^{\prime}, t^{\prime}\right)$, clearly if $\left(f^{\prime}, t^{\prime}\right)$ individually dominates $(f, t)$, then it also collectively dominates $(f, t)$. Theorem 3.4 shows that the reverse implication however does not need to hold, even if we limit ourselves to special types of mechanisms. That is, the fact that $\left(f^{\prime}, t^{\prime}\right)$ collectively dominates $(f, t)$ does not imply that $\left(f^{\prime}, t^{\prime}\right)$ individually dominates $(f, t)$.

Given a set $Z$ of payment-based mechanisms, individual and collective dominance induce two partial orders on $Z$, and we are interested in studying the maximal elements with respect to these partial orders. A maximal element with respect to the first partial order will be called an individually undominated mechanism, i.e., it is a mechanism that is not individually dominated by any other mechanism in $Z$. A maximal element for the second partial order will be called a collectively undominated mechanism, i.e., it is a mechanism that is not collectively dominated by any other mechanism in $Z$. The maximal elements with respect to the two partial orders may differ and in particular, the notion of collectively undominated mechanisms is generally a stronger notion. Clearly, if $\left(f^{\prime}, t^{\prime}\right) \in Z$ is collectively undominated, then it is also individually undominated. The reverse may not be true, examples of which are provided in Section 4.2.

If we focus on the same decision function $f$, then individual and collective dominance are strictly due to the difference of the payment functions. Hence, $\left(f, t^{\prime}\right)$ individually dominates $(f, t)$ (or simply $t^{\prime}$ individually dominates $t$ ) if and only if

- for all $\theta \in \Theta$ and all $i \in\{1, \ldots, n\}, t_{i}(\theta) \geq t_{i}^{\prime}(\theta)$, and

- for some $\theta \in \Theta$ and some $i \in\{1, \ldots, n\}, t_{i}(\theta)>t_{i}^{\prime}(\theta)$,

and $t^{\prime}$ collectively dominates $t$ if

- for all $\theta \in \Theta, \sum_{i=1}^{n} t_{i}(\theta) \geq \sum_{i=1}^{n} t_{i}^{\prime}(\theta)$, and

- for some $\theta \in \Theta, \sum_{i=1}^{n} t_{i}(\theta)>\sum_{i=1}^{n} t_{i}^{\prime}(\theta)$.

We now define two transformations on payment-based mechanisms originating from the same decision function. Both transformations build upon the surplus-guarantee concept (Cavallo, 2006) for the specific case of the VCG mechanism ${ }^{6}$.

Consider a payment-based mechanism $(f, t)$. Given $\theta=\left(\theta_{1}, \ldots, \theta_{n}\right)$, let $T(\theta)$ be the total amount of payments, i.e., $T(\theta):=\sum_{i=1}^{n} t_{i}(\theta)$. For each $i \in\{1, \ldots, n\}$ let

$$
S_{i}^{B C G C}\left(\theta_{-i}\right):=\inf _{\theta_{i}^{\prime} \in \Theta_{i}} T\left(\theta_{i}^{\prime}, \theta_{-i}\right) .
$$

In other words, $S_{i}^{B C G C}\left(\theta_{-i}\right)$ is the surplus guarantee independent of the report of agent $i$. We then define the payment-based mechanism $\left(f, t^{B C G C}\right)$ by setting for $i \in\{1, \ldots, n\}$

$$
t_{i}^{B C G C}(\theta):=t_{i}(\theta)-\frac{S_{i}^{B C G C}\left(\theta_{-i}\right)}{n} .
$$

6. The first transformation was originally defined by Bailey (1997) and Cavallo (2006) for the specific case of the VCG mechanism and by Guo and Conitzer (2008b) for non-deficit Groves mechanisms. We call it the $\boldsymbol{B C G C}$ transformation after the authors of these papers (Bailey, Cavallo, Guo, Conitzer). 
Also, for a fixed agent $j$, we define the payment-based mechanism $\left(f, t^{B C G C(j)}\right)$ by setting for $i \in\{1, \ldots, n\}$

$$
t_{i}^{B C G C(j)}(\theta):= \begin{cases}t_{i}(\theta)-S_{i}^{B C G C}\left(\theta_{-i}\right) & \text { if } i=j \\ t_{i}(\theta) & \text { if } i \neq j\end{cases}
$$

After the first transformation (from $(f, t)$ to $\left(f, t^{B C G C}\right)$ ), every agent receives an additional $^{7}$ amount of $\frac{1}{n}$ times the surplus guarantee independent of his own type. During the second transformation (from $(f, t)$ to $\left(f, t^{B C G C(j)}\right)$, agent $j$ is chosen to be the only agent who receives an additional amount. This additional amount equals the entirety of the surplus guarantee independent of $j$ 's own type. For both transformations the agents' additional payments are independent of their own types, thus the strategy-proofness is maintained: if $(f, t)$ is strategy-proof, then so are $\left(f, t^{B C G C}\right)$ and $\left(f, t^{B C G C(j)}\right)$ for all $j$.

The following observations generalize some of the earlier results by Bailey (1997) and Cavallo (2006).

\section{Proposition 2.3}

(i) Each payment-based mechanism of the form $t^{B C G C}$ is non-deficit.

(ii) If $t$ is non-deficit, then either $t$ and $t^{B C G C}$ coincide or $t^{B C G C}$ individually (and hence also collectively) dominates $t$.

Proof. (i) For all $\theta$ and $i \in\{1, \ldots, n\}$ we have $T(\theta) \geq S_{i}^{B C G C}\left(\theta_{-i}\right)$, so

$$
\begin{aligned}
T^{B C G C}(\theta) & =\sum_{i=1}^{n} t_{i}^{B C G C}(\theta)=T(\theta)-\sum_{i=1}^{n} \frac{S_{i}^{B C G C}\left(\theta_{-i}\right)}{n} \\
& =\sum_{i=1}^{n} \frac{T(\theta)-S_{i}^{B C G C}\left(\theta_{-i}\right)}{n} \geq 0 .
\end{aligned}
$$

(ii) If $t$ is non-deficit, then for all $\theta$ and all $i \in\{1, \ldots, n\}$ we have $S_{i}^{B C G C}\left(\theta_{-i}\right) \geq 0$, and hence $t_{i}^{B C G C}(\theta) \leq t_{i}(\theta)$.

The same claims hold for $t^{B C G C(j)}$ for $j \in\{1, \ldots, n\}$, with equally simple proofs.

\section{Groves Mechanisms}

We first briefly review Groves mechanisms.

\subsection{Preliminaries}

Recall that a Groves (1973) Mechanism is a payment-based mechanism $(f, t)$ such that the following hold ${ }^{8}$ :

7. Receiving an additional positive amount means paying less and receiving an additional negative amount means paying more.

8. Here and below $\sum_{j \neq i}$ is a shorthand for the summation over all $j \in\{1, \ldots, n\}, j \neq i$. 
- $f(\theta) \in \arg \max _{d \in D} \sum_{i=1}^{n} v_{i}\left(d, \theta_{i}\right)$, i.e., the chosen outcome maximizes the allocation welfare (the agents' total valuation),

- $t_{i}: \Theta \rightarrow \mathbb{R}$ is defined by $t_{i}(\theta):=h_{i}\left(\theta_{-i}\right)-g_{i}(\theta)$, where

- $g_{i}(\theta):=\sum_{j \neq i} v_{j}\left(f(\theta), \theta_{j}\right)$,

- $h_{i}: \Theta_{-i} \rightarrow \mathbb{R}$ is an arbitrary function.

So $g_{i}(\theta)$ represents the allocation welfare from the decision $f(\theta)$ with agent $i$ ignored. Recall now the following crucial result (see, e.g., Mas-Colell et al., 1995).

Groves Theorem (Groves, 1973) Every Groves mechanism is efficient and strategyproof.

For several decision problems, the only efficient and strategy-proof payment-based mechanisms are Groves mechanisms. This is implied by a general result by Holmström (1979), which covers the two domains that we consider in Sections 5 and 6, and explains our focus on Groves mechanisms. Hence from now on, we use the term "mechanism" to refer to a Groves mechanism.

Focusing on the set of non-deficit Groves mechanisms, individually (respectively, collectively) undominated mechanisms are the mechanisms from this set that are not individually (respectively, collectively) dominated by any other non-deficit Groves mechanism. As mentioned earlier, no matter which domain and which set of mechanisms we consider, collective undominance always implies individual undominance. In Section 4.2 we show two examples of single-item auction scenarios, where collective undominance is strictly stronger than individual undominance, for non-deficit Groves mechanisms. That is, there exists an individually undominated non-deficit Groves mechanism that is collectively dominated.

Recall that a special Groves mechanism, called the VCG or Clarke (1971) mechanism, is obtained using ${ }^{9}$

$$
h_{i}\left(\theta_{-i}\right):=\max _{d \in D} \sum_{j \neq i} v_{j}\left(d, \theta_{j}\right)
$$

In this case

$$
t_{i}(\theta):=\max _{d \in D} \sum_{j \neq i} v_{j}\left(d, \theta_{j}\right)-\sum_{j \neq i} v_{j}\left(f(\theta), \theta_{j}\right),
$$

which shows that the VCG mechanism is pay-only.

In what follows we introduce a slightly different notation to describe Groves mechanisms, that makes the rest of our presentation more convenient. First, we denote the payment function $t_{i}$ of the VCG mechanism by $V C G_{i}$. Note now that each Groves mechanism $(f, t)$ can be defined in terms of the VCG mechanism by setting $t_{i}(\theta):=V C G_{i}(\theta)-r_{i}\left(\theta_{-i}\right)$, where $r_{i}: \Theta_{-i} \rightarrow \mathbb{R}$ is some function of $\theta_{-i}$. We refer then to $\mathbf{r}:=\left(r_{1}, \ldots, r_{n}\right)$ as a redistribution function. Hence each Groves mechanism can be identified with a redistribution function $\mathbf{r}$ and can be viewed as the VCG mechanism combined with a redistribution. That is, under $\mathbf{r}$ the agents first participate in the VCG mechanism. Then, on top of that, agent $i$ also

9. Here and below, whenever $D$ is not a finite set, in order to ensure that the considered maximum exists, we assume that $f$ is continuous, and so is $v_{i}$ for each $i$, and also that the set $D$ and all $\Theta_{i}$ are compact subsets of some $\mathbb{R}^{k}$. 
receives a redistribution amount equal to $r_{i}\left(\theta_{-i}\right)$. By definition, a Groves mechanism $\mathbf{r}$ is non-deficit iff $\sum_{i=1}^{n} V C G_{i}(\theta) \geq \sum_{i=1}^{n} r_{i}\left(\theta_{-i}\right)$ for all $\theta \in \Theta$.

\subsection{Dominance Relations}

Using the new notation above, individual and collective dominance (among non-deficit Groves mechanisms) can be described as follows:

Definition 3.1 A non-deficit Groves mechanism $\mathbf{r}^{\prime}$ individually dominates another nondeficit Groves mechanism $\mathbf{r}$ if

- for all $i$ and all $\theta, r_{i}^{\prime}\left(\theta_{-i}\right) \geq r_{i}\left(\theta_{-i}\right)$,

- for some $i$ and some $\theta, r_{i}^{\prime}\left(\theta_{-i}\right)>r_{i}\left(\theta_{-i}\right)$.

Definition 3.2 A non-deficit Groves mechanism $\mathbf{r}^{\prime}$ collectively dominates another nondeficit Groves mechanism $\mathbf{r}$ if

- for all $\theta, \sum_{i} r_{i}^{\prime}\left(\theta_{-i}\right) \geq \sum_{i} r_{i}\left(\theta_{-i}\right)$,

- for some $\theta, \sum_{i} r_{i}^{\prime}\left(\theta_{-i}\right)>\sum_{i} r_{i}\left(\theta_{-i}\right)$.

We now consider the mechanism that results from applying the BCGC transformation to the VCG mechanism. We refer to this as the Bailey-Cavallo mechanism or simply the BC mechanism (Bailey, 1997; Cavallo, 2006). The VCG mechanism is characterized by the constant redistribution function $\mathbf{r}^{\mathbf{V C G}}=(0,0, \ldots, 0)$. After the BCGC transformation, every agent $i$ receives an additional amount of $\frac{1}{n}$ times the surplus guarantee $S_{i}^{B C G C}\left(\theta_{-i}\right)$, independent of his own type. That is, the BC mechanism is also a Groves mechanism, and its redistribution function is

$$
\mathbf{r}^{\mathbf{B C}}=\left(\frac{1}{n} S_{1}^{B C G C}, \frac{1}{n} S_{2}^{B C G C}, \ldots, \frac{1}{n} S_{n}^{B C G C}\right) .
$$

Let $\theta^{\prime}:=\left(\theta_{1}, \ldots, \theta_{i-1}, \theta_{i}^{\prime}, \theta_{i+1}, \ldots, \theta_{n}\right)$. Then starting from the VCG mechanism, we have

$$
S_{i}^{B C G C}\left(\theta_{-i}\right)=\inf _{\theta_{i}^{\prime} \in \Theta_{i}} \sum_{k=1}^{n}\left[\max _{d \in D} \sum_{j \neq k} v_{j}\left(d, \theta_{j}^{\prime}\right)-\sum_{j \neq k} v_{j}\left(f\left(\theta^{\prime}\right), \theta_{j}^{\prime}\right)\right],
$$

that is,

$$
S_{i}^{B C G C}\left(\theta_{-i}\right)=\inf _{\theta_{i}^{\prime} \in \Theta_{i}}\left[\sum_{k=1}^{n} \max _{d \in D} \sum_{j \neq k} v_{j}\left(d, \theta_{j}^{\prime}\right)-(n-1) \sum_{k=1}^{n} v_{k}\left(f\left(\theta^{\prime}\right), \theta_{k}^{\prime}\right)\right]
$$

In many settings, we have that for all $\theta$ and for all $i, S_{i}^{B C G C}\left(\theta_{-i}\right)=0$, and consequently the VCG and BC mechanisms coincide (e.g., see Proposition 6.1). Whenever they do not, by Proposition 2.3(ii), BC individually and collectively dominates VCG. This is the case for the single-item auction, as it can be seen that there $S_{i}^{B C G C}\left(\theta_{-i}\right)=\left[\theta_{-i}\right]_{2}$, where $\left[\theta_{-i}\right]_{2}$ is the second-highest bid among bids other than agent $i$ 's own bid. 


\subsection{Anonymous Groves Mechanisms}

Some of the proofs of our main results are obtained by arguing first about a special class of Groves mechanisms, called anonymous Groves mechanisms. We provide here some results about this class that we will utilize in later sections. We call a function $f: A^{n} \rightarrow B$ permutation independent if for all permutations $\pi$ of $\{1, \ldots, n\}, f=f \circ \pi$. Following Moulin (1986), we call a Groves mechanism $\mathbf{r}=\left(r_{1}, \ldots, r_{n}\right)$ anonymous $^{10}$ if

- all type sets $\Theta_{i}$ are equal,

- all functions $r_{i}$ coincide and each of them is permutation independent.

Hence, an anonymous Groves mechanism is uniquely determined by a single function $r$ : $\Theta^{n-1} \rightarrow \mathbb{R}$

In general, the VCG mechanism is not anonymous. But it is anonymous when all the type sets are equal and all the initial utility functions $v_{i}$ coincide. This is the case in the two domains that we consider in later sections.

For any $\theta \in \Theta$ and any permutation $\pi$ of $\{1, \ldots, n\}$ we define $\theta^{\pi} \in \Theta$ by letting

$$
\theta_{i}^{\pi}:=\theta_{\pi^{-1}(i)} \cdot
$$

Denote by $\Pi(k)$ the set of all permutations of the set $\{1, \ldots, k\}$. Given a Groves mechanism $\mathbf{r}:=\left(r_{1}, \ldots, r_{n}\right)$ for which the type set $\Theta_{i}$ is the same for every agent (and equal to some set $\Theta_{0}$ ), we construct now a function $r^{\prime}: \Theta_{0}^{n-1} \rightarrow \mathbb{R}$, following Moulin (1986), by setting

$$
r^{\prime}(x):=\sum_{j=1}^{n} \frac{\sum_{\pi \in \Pi(n-1)} r_{j}\left(x^{\pi}\right)}{n !},
$$

where $x^{\pi}$ is defined analogously to $\theta^{\pi}$.

Note that $r^{\prime}$ is permutation independent, so $r^{\prime}$ is an anonymous Groves mechanism. The following lemma, which can be of independent interest, shows that some of the properties of $\mathbf{r}$ transfer to $r^{\prime}$.

Lemma 3.3 Consider a Groves mechanism $r$ and the corresponding anonymous Groves mechanism $r^{\prime}$. Let $V C G(\theta):=\sum_{i=1}^{n} V C G_{i}(\theta)$, and suppose that the $V C G$ function is permutation independent. Then:

(i) If $\boldsymbol{r}$ is non-deficit, so is $r^{\prime}$.

(ii) If an anonymous Groves mechanism $r^{0}$ is collectively dominated by $r$, then it is collectively dominated by $r^{\prime}$.

Proof. For all $\theta \in \Theta$ we have

$$
\sum_{i=1}^{n} r_{i}^{\prime}\left(\theta_{-i}\right)=\frac{\sum_{i=1}^{n} \sum_{\pi \in \Pi(n-1)} \sum_{j=1}^{n} r_{j}\left(\left(\theta_{-i}\right)^{\pi}\right)}{n !}=
$$

10. Our definition is slightly different than the one introduced by Moulin (1986) in that no conditions are put on the utility functions and the permutation independence refers only to the redistribution function. 


$$
\frac{\sum_{i=1}^{n} \sum_{\pi \in \Pi(n)} r_{i}\left(\theta_{-i}^{\pi}\right)}{n !}
$$

where the last equality holds since in both terms we aggregate over all applications of all $r_{i}$ functions to all permutations of $n-1$ elements of $\theta$.

Let $t$ and $t^{\prime}$ be the payment functions of the mechanisms $\mathbf{r}$ and $r^{\prime}$, respectively. We have

$$
\sum_{i=1}^{n} t_{i}^{\prime}(\theta)=V C G(\theta)-\sum_{i=1}^{n} r_{i}^{\prime}\left(\theta_{-i}\right)
$$

and for all $\pi \in \Pi(n)$

$$
\sum_{i=1}^{n} t_{i}\left(\theta^{\pi}\right)=\operatorname{VCG}\left(\theta^{\pi}\right)-\sum_{i=1}^{n} r_{i}\left(\theta_{-i}^{\pi}\right) .
$$

Hence by the assumption about $V C G(\theta)$ it follows that

$$
\sum_{i=1}^{n} t_{i}^{\prime}(\theta)=\frac{\sum_{\pi \in \Pi(n)} \sum_{i=1}^{n} t_{i}\left(\theta^{\pi}\right)}{n !}
$$

(i) is now an immediate consequence of (2).

To prove $(i i)$ let $t^{0}$ be the payment function of $r^{0} . \mathbf{r}$ collectively dominates $r^{0}$, so for all $\theta \in \Theta$ and all $\pi \in \Pi(n)$

$$
\sum_{i=1}^{n} t_{i}\left(\theta^{\pi}\right) \leq \sum_{i=1}^{n} t_{i}^{0}\left(\theta^{\pi}\right)
$$

with at least one inequality strict. Hence for all $\theta \in \Theta$

$$
\frac{\sum_{\pi \in \Pi(n)} \sum_{i=1}^{n} t_{i}\left(\theta^{\pi}\right)}{n !} \leq \frac{\sum_{\pi \in \Pi(n)} \sum_{i=1}^{n} t_{i}^{0}\left(\theta^{\pi}\right)}{n !}
$$

with at least one inequality strict.

But the fact that $r^{0}$ is anonymous and the assumption about $V C G(\theta)$ imply that for all $\theta \in \Theta$ and all permutations $\pi$ of $\{1, \ldots, n\}$

$$
\sum_{i=1}^{n} t_{i}^{0}\left(\theta^{\pi}\right)=\sum_{i=1}^{n} t_{i}^{0}(\theta)
$$

so by (2) and the above inequality, we have that for all $\theta \in \Theta$

$$
\sum_{i=1}^{n} t_{i}^{\prime}(\theta) \leq \sum_{i=1}^{n} t_{i}^{0}(\theta)
$$

with at least one inequality strict.

The assumption in Lemma 3.3 of permutation independence of $V C G(\theta)$ is satisfied in both of the domains that we consider in Sections 5 and 6 . So item (ii) states that if a Groves mechanism considered in the sequel is not collectively undominated, then it is collectively dominated by an anonymous Groves mechanism.

We now prove that for a large class of Groves mechanisms that includes the ones we study in the sequel the introduced relations of dominance differ. 
Theorem 3.4 Suppose $n \geq 3$. Assume that the sets of types $\Theta_{i}$ are all equal to the set $\Theta_{0}$ which contains at least $n-1$ elements. Then two non-deficit anonymous Groves mechanisms $r$ and $r^{\prime}$ exist such that $r$ collectively dominates $r^{\prime}$ but $r$ does not individually dominate $r^{\prime}$.

Proof. Fix a non-deficit anonymous Groves mechanism determined by a permutation independent function $r: \Theta_{0}^{n-1} \rightarrow \mathbb{R}$.

Let $a_{1}, \ldots, a_{n-1}$ be arbitrary different elements of $\Theta_{0}$. Define a permutation independent function $q: \Theta_{0}^{n-1} \rightarrow \mathbb{R}$ by putting

$$
q(x):=\left\{\begin{aligned}
-1 & \text { if } x \text { is a permutation of }\left(a_{1}, \ldots, a_{n-1}\right) \\
2 & \text { otherwise }
\end{aligned}\right.
$$

Then for each $\theta \in \Theta_{0}^{n}$ at most two of its subsequences $\theta_{-i}$ may form a permutation of $\left(a_{1}, \ldots, a_{n-1}\right)$. But $n \geq 3$, so for all $\theta \in \Theta, \sum_{i=1}^{n} q\left(\theta_{-i}\right) \geq 0$. This implies that the anonymous Groves mechanism determined by the function $r^{\prime}:=r-q$ is non-deficit.

Trivially, the sum of payments under $r$ is less than or equal to the sum of payments under $r^{\prime}$, since $r$ redistributes more money than $r^{\prime}$. Moreover for some $\theta \in \Theta$, for instance $\theta=\left(a_{1}, \ldots, a_{1}\right)$, we have $\sum_{i=1}^{n} q\left(\theta_{-i}\right)>0$. Finally, by definition, $q\left(a_{1}, \ldots, a_{n-1}\right)=-1$.

These imply that $r^{\prime}$ is collectively dominated by $r$ but is not individually dominated by $r$.

\section{Individually Undominated Mechanisms: Characterization and Algorithmic Techniques for General Domains}

In this section, we focus on individually undominated non-deficit Groves mechanisms.

\subsection{Non-deficit Groves Mechanisms}

We start with a characterization of non-deficit Groves mechanisms. Recall first that for a type profile $\theta$, we denote by $V C G(\theta)$ the total VCG payment, $\sum_{i=1}^{n} V C G_{i}(\theta)$.

Proposition 4.1 A Groves mechanism $\mathbf{r}$ is non-deficit if and only if for all $i$ and all $\theta$,

$$
r_{i}\left(\theta_{-i}\right) \leq \inf _{\theta_{i}^{\prime} \in \Theta_{i}}\left\{V C G\left(\theta_{i}^{\prime}, \theta_{-i}\right)-\sum_{j \neq i} r_{j}\left(\theta_{-j}^{\prime}\right)\right\}
$$

Here, $\theta_{-j}^{\prime}$ are the reported types of the agents other than $j$ when $\theta_{i}$ is replaced by $\theta_{i}^{\prime}$.

Proof. We first prove the "if" direction. For any $i$ and $\theta$, Equation 3 implies that $r_{i}\left(\theta_{-i}\right) \leq$ $V C G\left(\theta_{i}^{\prime}, \theta_{-i}\right)-\sum_{j \neq i} r_{j}\left(\theta_{-j}^{\prime}\right)$ for any $\theta_{i}^{\prime} \in \Theta_{i}$. If we let $\theta_{i}^{\prime}=\theta_{i}$, we obtain $\sum_{j} r_{j}\left(\theta_{-j}\right) \leq$ $V C G\left(\theta_{i}, \theta_{-i}\right)=\sum_{i} V C G_{i}(\theta)$. Thus, the non-deficit property holds.

We now prove the "only if" direction. To ensure the non-deficit property, for any $i$, any $\theta_{-i}$, and any $\theta_{i}^{\prime}$, we must have $r_{i}\left(\theta_{-i}\right)+\sum_{j \neq i} r_{j}\left(\theta_{-j}^{\prime}\right) \leq V C G\left(\theta_{i}^{\prime}, \theta_{-i}\right)$, or equivalently $r_{i}\left(\theta_{-i}\right) \leq V C G\left(\theta_{i}^{\prime}, \theta_{-i}\right)-\sum_{j \neq i} r_{j}\left(\theta_{-j}^{\prime}\right)$. Since $\theta_{i}^{\prime}$ is arbitrary, Equation 3 follows.

By replacing the " $\leq$ " in Equation 3 by "=", we get a characterization of individually undominated non-deficit Groves mechanisms. 
Theorem 4.2 A Groves mechanism $\mathbf{r}$ is non-deficit and individually undominated if and only if for all $i$ and all $\theta$,

$$
r_{i}\left(\theta_{-i}\right)=\inf _{\theta_{i}^{\prime} \in \Theta_{i}}\left\{V C G\left(\theta_{i}^{\prime}, \theta_{-i}\right)-\sum_{j \neq i} r_{j}\left(\theta_{-j}^{\prime}\right)\right\}
$$

Here, $\theta_{-j}^{\prime}$ are the reported types of the agents other than $j$ when $\theta_{i}$ is replaced by $\theta_{i}^{\prime}$.

Proof. We prove the "if" direction first. Any Groves mechanism $\mathbf{r}$ that satisfies Equation 4 is non-deficit by Proposition 4.1. Now suppose that $\mathbf{r}$ is individually dominated, that is, there exists another non-deficit Groves mechanism $\mathbf{r}^{\prime}$ such that for all $i$ and $\theta_{-i}$, we have $r_{i}^{\prime}\left(\theta_{-i}\right) \geq r_{i}\left(\theta_{-i}\right)$, and for some $i$ and $\theta_{-i}$, we have $r_{i}^{\prime}\left(\theta_{-i}\right)>r_{i}\left(\theta_{-i}\right)$. For the $i$ and $\theta_{-i}$ that make this inequality strict, we have

$$
\begin{aligned}
r_{i}^{\prime}\left(\theta_{-i}\right)> & r_{i}\left(\theta_{-i}\right)=\inf _{\theta_{i}^{\prime} \in \Theta_{i}}\left\{V C G\left(\theta_{i}^{\prime}, \theta_{-i}\right)-\sum_{j \neq i} r_{j}\left(\theta_{-j}^{\prime}\right)\right\} \\
& \geq \inf _{\theta_{i}^{\prime} \in \Theta_{i}}\left\{V C G\left(\theta_{i}^{\prime}, \theta_{-i}\right)-\sum_{j \neq i} r_{j}^{\prime}\left(\theta_{-j}^{\prime}\right)\right\},
\end{aligned}
$$

which contradicts with the fact that $\mathbf{r}^{\prime}$ must satisfy Equation 3. It follows that $\mathbf{r}$ is individually undominated.

Now we prove the "only if" direction. Suppose Equation 4 is not satisfied. Then, there exists some $i$ and $\theta_{-i}$ such that $r_{i}\left(\theta_{-i}\right)<\inf _{\theta_{i}^{\prime} \in \Theta_{i}}\left\{V C G\left(\theta_{i}^{\prime}, \theta_{-i}\right)-\sum_{j \neq i} r_{j}\left(\theta_{-j}^{\prime}\right)\right\}$. Let $a=\inf _{\theta_{i}^{\prime} \in \Theta_{i}}\left\{V C G\left(\theta_{i}^{\prime}, \theta_{-i}\right)-\sum_{j \neq i} r_{j}\left(\theta_{-j}^{\prime}\right)\right\}-r_{i}\left(\theta_{-i}\right)$ (so that $a>0$ ), and let $\mathbf{r}^{\prime}$ be the same as $\mathbf{r}$, except that for the aforementioned $i$ and $\theta_{-i}, r_{i}^{\prime}\left(\theta_{-i}\right)=r_{i}\left(\theta_{-i}\right)+a$. To show that this does not break the non-deficit constraint, consider any type vector $\left(\theta_{i}, \theta_{-i}\right)$ where $i$ and $\theta_{-i}$ are the same as before (that is, any type profile that is affected). Then,

$$
\begin{aligned}
r_{i}^{\prime}\left(\theta_{-i}\right)=a & +r_{i}\left(\theta_{-i}\right)=\inf _{\theta_{i}^{\prime} \in \Theta_{i}}\left\{V C G\left(\theta_{i}^{\prime}, \theta_{-i}\right)-\sum_{j \neq i} r_{j}\left(\theta_{-j}^{\prime}\right)\right\} \\
= & \inf _{\theta_{i}^{\prime} \in \Theta_{i}}\left\{V C G\left(\theta_{i}^{\prime}, \theta_{-i}\right)-\sum_{j \neq i} r_{j}^{\prime}\left(\theta_{-j}^{\prime}\right)\right\} .
\end{aligned}
$$

Thus, by Proposition 4.1, $\mathbf{r}^{\prime}$ is non-deficit. This contradicts that $\mathbf{r}$ is individually undominated. Hence, Equation 4 must hold.

We now give an example of an individually undominated mechanism.

Example 4.3 Consider a single-item auction with $n \geq 3$ agents. Agent $i$ bids $\theta_{i} \in[0, \infty)$. Let $[\theta]_{j}$ be the $j$ th highest type from the type profile $\theta$. Let us consider the anonymous Groves mechanism characterized by $r\left(\theta_{-i}\right)=\frac{1}{n}\left[\theta_{-i}\right]_{2}$. That is, under this mechanism, besides paying the VCG payment, every agent receives $\frac{1}{n}$ times the second highest other bid. In fact, this mechanism is the $\mathrm{BC}$ mechanism for single-item auctions. To show that $r$ is individually undominated, it suffices to show Equation 4 is satisfied. We first observe that for every agent, the second highest other bid is no more than the second highest bid, 
which equals the total VCG payment. That is, $r$ is non-deficit. Hence, Equation 3 holds for all agents and all type profiles. Moreover, for every type profile $\theta$, by setting $\theta_{i}^{\prime}=\left[\theta_{-i}\right]_{2}$, we can verify that Equation 4 holds. It follows that the $\mathrm{BC}$ mechanism is individually undominated for single-item auctions.

In what follows, we first show two examples of single-item auction scenarios, where collective undominance is strictly stronger than individual undominance for non-deficit Groves mechanisms. We then propose two techniques for generating individually undominated mechanisms starting from known individually dominated mechanisms (if the initial mechanism is already individually undominated, then the techniques will return the same mechanism). One technique immediately produces an individually undominated mechanism. However, it does not preserve anonymity. The second technique preserves anonymity, and after repeated applications the result converges to an individually undominated mechanism. We emphasize that we can start with any non-deficit Groves mechanism, including the BC mechanism, the Worst-Case Optimal mechanism (Guo \& Conitzer, 2009), the Optimal-inExpectation Linear mechanisms (Guo \& Conitzer, 2010), and the VCG mechanism.

\subsection{Collective Undominance is Strictly Stronger than Individual Undominance}

We use two examples to show that collective undominance is, in general, strictly stronger than individual undominance.

Example 4.4 Consider a single-item auction with 4 agents. We assume that for each agent, the set of allowed types is the same, namely, integers from 0 to 3 . Here, the VCG mechanism is just the second-price auction.

Let us consider the following two anonymous non-deficit Groves mechanisms, which are computer-generated for differentiating collective undominance and individual undominance.

Mechanism 1: $r\left(\theta_{-i}\right)=r\left(\left[\theta_{-i}\right]_{1},\left[\theta_{-i}\right]_{2},\left[\theta_{-i}\right]_{3}\right)$, and the function $r$ is given in Table 1 . $\left(\left[\theta_{-i}\right]_{j}\right.$ is the $j$ th highest type among types other than $i$ 's own type.)

Mechanism 2: $r^{\prime}\left(\theta_{-i}\right)=r^{\prime}\left(\left[\theta_{-i}\right]_{1},\left[\theta_{-i}\right]_{2},\left[\theta_{-i}\right]_{3}\right)$, and the function $r^{\prime}$ is given in Table 1 .

With the above characterization, we have that mechanism 2 collectively dominates mechanism 1: for example, for the type profile $(3,2,2,2), \sum_{i} r\left(\theta_{-i}\right)=1 / 2<1=\sum_{i} r^{\prime}\left(\theta_{-i}\right)$. On the other hand, mechanism 2 does not individually dominate mechanism 1: for example, $r(3,3,2)=1>5 / 6=r^{\prime}(3,3,2)$. In fact, based on the characterization of individually undominated non-deficit Groves mechanisms (Theorem 4.2), we are able to show that mechanism 1 is individually undominated.

Example 4.5 Consider a single-item auction with 5 agents. We assume that for each agent, the set of allowed types is $[0, \infty)$. Here, the VCG mechanism is just the second-price auction.

Let us consider the following two anonymous non-deficit Groves mechanisms:

\section{Mechanism 1:}




\begin{tabular}{|c|c|c|c||c|c|c|c|}
\hline $\mathbf{r}(\mathbf{0}, \mathbf{0}, \mathbf{0})$ & 0 & $\mathbf{r}^{\prime}(\mathbf{0}, \mathbf{0}, \mathbf{0})$ & 0 & $\mathbf{r}(\mathbf{3}, \mathbf{0}, \mathbf{0})$ & 0 & $\mathbf{r}^{\prime}(\mathbf{3}, \mathbf{0}, \mathbf{0})$ & 0 \\
\hline $\mathbf{r}(\mathbf{1}, \mathbf{0}, \mathbf{0})$ & 0 & $\mathbf{r}^{\prime}(\mathbf{1}, \mathbf{0}, \mathbf{0})$ & 0 & $\mathbf{r}(\mathbf{3}, \mathbf{1}, \mathbf{0})$ & $1 / 4$ & $\mathbf{r}^{\prime}(\mathbf{3}, \mathbf{1}, \mathbf{0})$ & $1 / 4$ \\
\hline $\mathbf{r}(\mathbf{1}, \mathbf{1}, \mathbf{0})$ & $1 / 4$ & $\mathbf{r}^{\prime}(\mathbf{1}, \mathbf{1}, \mathbf{0})$ & $1 / 4$ \\
\hline $\mathbf{r}(\mathbf{1}, \mathbf{1}, \mathbf{1})$ & $1 / 4$ & $\mathbf{r}^{\prime}(\mathbf{1}, \mathbf{1}, \mathbf{1})$ & $1 / 4$ & $\mathbf{r}(\mathbf{3}, \mathbf{1}, \mathbf{1})$ & 0 & $\mathbf{r}^{\prime}(\mathbf{3}, \mathbf{1}, \mathbf{1})$ & $1 / 4$ \\
\hline $\mathbf{r}(\mathbf{2}, \mathbf{0}, \mathbf{0})$ & 0 & $\mathbf{r}^{\prime}(\mathbf{2}, \mathbf{0}, \mathbf{0})$ & 0 & $\mathbf{r}(\mathbf{3}, \mathbf{2}, \mathbf{0})$ & $2 / 3$ & $\mathbf{r}^{\prime}(\mathbf{3}, \mathbf{2}, \mathbf{0})$ & $2 / 3$ \\
\hline $\mathbf{r}(\mathbf{2}, \mathbf{1}, \mathbf{0})$ & $1 / 12$ & $\mathbf{r}^{\prime}(\mathbf{2}, \mathbf{1}, \mathbf{0})$ & $7 / 24$ & $\mathbf{r}, \mathbf{1})$ & 1 & $\mathbf{r}^{\prime}(\mathbf{3}, \mathbf{2}, \mathbf{1})$ & $19 / 24$ \\
\hline $\mathbf{r}(\mathbf{2}, \mathbf{1}, \mathbf{1})$ & 0 & $\mathbf{r}^{\prime}(\mathbf{2}, \mathbf{1}, \mathbf{1})$ & $1 / 6$ & $\mathbf{r}, \mathbf{2})$ & 0 & $\mathbf{r}^{\prime}(\mathbf{3}, \mathbf{2}, \mathbf{2})$ & $1 / 6$ \\
\hline $\mathbf{r}(\mathbf{2}, \mathbf{2}, \mathbf{0})$ & $1 / 2$ & $\mathbf{r}^{\prime}(\mathbf{2}, \mathbf{2}, \mathbf{0})$ & $1 / 2$ & $\mathbf{r}(\mathbf{3}, \mathbf{0})$ & $2 / 3$ & $\mathbf{r}^{\prime}(\mathbf{3}, \mathbf{3}, \mathbf{0})$ & $5 / 6$ \\
\hline $\mathbf{r}(\mathbf{2}, \mathbf{2}, \mathbf{1})$ & 0 & $\mathbf{r}^{\prime}(\mathbf{2}, \mathbf{2}, \mathbf{1})$ & $1 / 4$ & 0 & $\mathbf{r}^{\prime}(\mathbf{3}, \mathbf{3}, \mathbf{1})$ & $7 / 12$ \\
\hline $\mathbf{r}(\mathbf{2}, \mathbf{2}, \mathbf{2})$ & $1 / 2$ & $\mathbf{r}^{\prime}(\mathbf{2}, \mathbf{2}, \mathbf{2})$ & $1 / 2$ & $\mathbf{r}(\mathbf{3}, \mathbf{3}, \mathbf{2})$ & 1 & $\mathbf{r}^{\prime}(\mathbf{3}, \mathbf{3}, \mathbf{2})$ & $5 / 6$ \\
\hline $\mathbf{r}(\mathbf{3}, \mathbf{3}, \mathbf{3})$ & 0 & $\mathbf{r}^{\prime}(\mathbf{3}, \mathbf{3}, \mathbf{3})$ & $1 / 2$ \\
\hline
\end{tabular}

Table 1: Computer-generated example mechanisms for differentiating collective undominance and individual undominance.

$r\left(\theta_{-i}\right)=0$ if all four types in $\theta_{-i}$ are identical.

$r\left(\theta_{-i}\right)=\left[\theta_{-i}\right]_{1} / 4$ if the highest three types in $\theta_{-i}$ are identical, and they are strictly higher than the lowest type in $\theta_{-i}$.

$r\left(\theta_{-i}\right)=\left[\theta_{-i}\right]_{1} / 6$ if the highest two types in $\theta_{-i}$ are identical, and they are strictly higher than the third highest type in $\theta_{-i}$.

$r\left(\theta_{-i}\right)=3\left[\theta_{-i}\right]_{2} / 16$ if the highest type in $\theta_{-i}$ is strictly higher than the second highest type in $\theta_{-i}$, and the second highest type in $\theta_{-i}$ is identical to the third highest type in $\theta_{-i}$. $r\left(\theta_{-i}\right)=\left[\theta_{-i}\right]_{2} / 5$ if the highest three types in $\theta_{-i}$ are all different.

Mechanism 2 (BC):

$r^{\prime}\left(\theta_{-i}\right)=\left[\theta_{-i}\right]_{2} / 5$.

With the above characterization, we have that mechanism 2 collectively dominates mechanism 1: for example, for the type profile $(3,2,2,2,2), \sum_{i} r\left(\theta_{-i}\right)=4 r(3,2,2,2)+$ $r(2,2,2,2)=3 / 2+0=3 / 2<\sum_{i} r^{\prime}\left(\theta_{-i}\right)=4 r^{\prime}(3,2,2,2)+r^{\prime}(2,2,2,2)=8 / 5+2 / 5=2$. On the other hand, mechanism 2 does not individually dominate mechanism 1: for example, $r(4,4,4,1)=1>4 / 5=r^{\prime}(4,4,4,1)$. In fact, based on the characterization of individually undominated non-deficit Groves mechanisms (Theorem 4.2), we are able to show that mechanism 1 is individually undominated.

\subsection{A Priority-Based Technique}

Given a non-deficit Groves mechanism $\mathbf{r}$ and a priority order over agents $\pi$, we can improve $\mathbf{r}$ into an individually undominated mechanism as follows:

1) Let $\pi:\{1, \ldots, n\} \rightarrow\{1, \ldots, n\}$ be a permutation representing the priority order. That is, $\pi(i)$ is agent $i$ 's priority value (the lower the value, the higher the priority). $\pi^{-1}(k)$ is then the agent with the $k$ th highest priority. The high-level idea of the priority-based technique is that we go over the agents one by one. For the first agent (the agent with the highest priority), we maximize his redistribution function subject to the constraint of 
Proposition 4.1. For later agents, we do the same, but take into consideration that earlier agents' redistribution functions have been updated. A priority order can be arbitrary. Generally, agents with high priorities benefit more from this technique, since for earlier agents, there is more room for improvement.

2) Let $i=\pi^{-1}(1)$, and update $r_{i}$ to

$$
r_{i}^{\pi}\left(\theta_{-i}\right)=\inf _{\theta_{i}^{\prime} \in \Theta_{i}}\left\{V C G\left(\theta_{i}^{\prime}, \theta_{-i}\right)-\sum_{\pi(j)>1} r_{j}\left(\theta_{-j}^{\prime}\right)\right\} .
$$

That is, the update ensures that at this point $\mathbf{r}^{\pi}$ satisfies Equation 4 for $i=\pi^{-1}(1)$.

It should be noted that during the above update, only the payment of agent $i=\pi^{-1}(1)$ is changed, and it is changed by

$$
\begin{gathered}
r_{i}^{\pi}\left(\theta_{-i}\right)-r_{i}\left(\theta_{-i}\right)=\inf _{\theta_{i}^{\prime} \in \Theta_{i}}\left\{V C G\left(\theta_{i}^{\prime}, \theta_{-i}\right)-\sum_{\pi(j)>1} r_{j}\left(\theta_{-j}^{\prime}\right)\right\}-r_{i}\left(\theta_{-i}\right) \\
=\inf _{\theta_{i}^{\prime} \in \Theta_{i}}\left\{V C G\left(\theta_{i}^{\prime}, \theta_{-i}\right)-\sum_{j} r_{j}\left(\theta_{-j}^{\prime}\right)\right\}=S_{i}^{B C G C}\left(\theta_{-i}\right) .
\end{gathered}
$$

That is, essentially, the above update amounts to applying the $B C G C(i)$ transformation on $\mathbf{r}$, where $i=\pi^{-1}(1)$.

3) We will now consider the remaining agents in turn, according to the order $\pi$. In the $k$ th step, we update $r_{i}\left(i=\pi^{-1}(k)\right)$ to

$$
r_{i}^{\pi}\left(\theta_{-i}\right)=\inf _{\theta_{i}^{\prime} \in \Theta_{i}}\left\{V C G\left(\theta_{i}^{\prime}, \theta_{-i}\right)-\sum_{\pi(j)>k} r_{j}\left(\theta_{-j}^{\prime}\right)-\sum_{\pi(j)<k} r_{j}^{\pi}\left(\theta_{-j}^{\prime}\right)\right\} .
$$

That is, the update ensures that at this point $\mathbf{r}^{\pi}$ satisfies Equation 4 when $i=\pi^{-1}(k)$. To avoid breaking the non-deficit property, when we make the update, we take the previous $k-1$ updates into account. For this update, what we are doing is essentially applying the $B C G C\left(\pi^{-1}(k)\right)$ transformation on the resulting mechanism from the previous update.

Overall, for every agent $i$,

$$
r_{i}^{\pi}\left(\theta_{-i}\right)=\inf _{\theta_{i}^{\prime} \in \Theta_{i}}\left\{V C G\left(\theta_{i}^{\prime}, \theta_{-i}\right)-\sum_{\pi(j)>\pi(i)} r_{j}\left(\theta_{-j}^{\prime}\right)-\sum_{\pi(j)<\pi(i)} r_{j}^{\pi}\left(\theta_{-j}^{\prime}\right)\right\} .
$$

The new mechanism $\mathbf{r}^{\pi}$ satisfies the following properties:

Proposition 4.6 For all $i$ and $\theta_{-i}, r_{i}^{\pi}\left(\theta_{-i}\right) \geq r_{i}\left(\theta_{-i}\right)$.

Proof. First consider $i=\pi^{-1}(1)$, the agent with the highest priority. For any $\theta_{-i}$, we have $r_{i}^{\pi}\left(\theta_{-i}\right)=\inf _{\theta_{i}^{\prime} \in \Theta_{i}}\left\{V C G\left(\theta_{i}^{\prime}, \theta_{-i}\right)-\sum_{j \neq i} r_{j}\left(\theta_{-j}^{\prime}\right)\right\}$. Since $\mathbf{r}$ is non-deficit, by Equation 3, we have $r_{i}\left(\theta_{-i}\right) \leq \inf _{\theta_{i}^{\prime} \in \Theta_{i}}\left\{V C G\left(\theta_{i}^{\prime}, \theta_{-i}\right)-\sum_{j \neq i} r_{j}\left(\theta_{-j}^{\prime}\right)\right\}$. Hence $r_{i}^{\pi}\left(\theta_{-i}\right) \geq r_{i}\left(\theta_{-i}\right)$. 
For any $i \neq \pi^{-1}(1), r_{i}^{\pi}\left(\theta_{-i}\right)$ equals

$$
r_{i}\left(\theta_{-i}\right)+\inf _{\theta_{i}^{\prime} \in \Theta_{i}}\left\{V C G\left(\theta_{i}^{\prime}, \theta_{-i}\right)-r_{i}\left(\theta_{-i}\right)-\sum_{\pi(j)>\pi(i)} r_{j}\left(\theta_{-j}^{\prime}\right)-\sum_{\pi(j)<\pi(i)} r_{j}^{\pi}\left(\theta_{-j}^{\prime}\right)\right\} .
$$

We must show that

$$
\inf _{\theta_{i}^{\prime} \in \Theta_{i}}\left\{V C G\left(\theta_{i}^{\prime}, \theta_{-i}\right)-r_{i}\left(\theta_{-i}\right)-\sum_{\pi(j)>\pi(i)} r_{j}\left(\theta_{-j}^{\prime}\right)-\sum_{\pi(j)<\pi(i)} r_{j}^{\pi}\left(\theta_{-j}^{\prime}\right)\right\} \geq 0 .
$$

Consider $p=\pi^{-1}(\pi(i)-1)$ (the agent immediately before $i$ in terms of priority). For any $\theta_{i}, \theta_{-i}$, we have

$$
\begin{gathered}
V C G\left(\theta_{i}, \theta_{-i}\right)-r_{i}\left(\theta_{-i}\right)-\sum_{\pi(j)>\pi(i)} r_{j}\left(\theta_{-j}\right)-\sum_{\pi(j)<\pi(i)} r_{j}^{\pi}\left(\theta_{-j}\right) \\
=V C G\left(\theta_{i}, \theta_{-i}\right)-\sum_{\pi(j)>\pi(p)} r_{j}\left(\theta_{-j}\right)-\sum_{\pi(j)<\pi(p)} r_{j}^{\pi}\left(\theta_{-j}\right)-r_{p}^{\pi}\left(\theta_{-p}\right) \\
\geq \inf _{\theta_{p}^{\prime} \in \Theta_{p}}\left\{V C G\left(\theta_{p}^{\prime}, \theta_{-p}\right)-\sum_{\pi(j)>\pi(p)} r_{j}\left(\theta_{-j}^{\prime}\right)-\sum_{\pi(j)<\pi(p)} r_{j}^{\pi}\left(\theta_{-j}^{\prime}\right)\right\}-r_{p}^{\pi}\left(\theta_{-p}\right)=0 .
\end{gathered}
$$

In the above inequality, $\theta_{-j}^{\prime}$ is the set of types reported by the agents other than $j$, when $\theta_{p}$ is replaced by $\theta_{p}^{\prime}$. Because $\theta_{i}$ is arbitrary, Equation 5 follows. Therefore, $r_{i}^{\pi}\left(\theta_{-i}\right) \geq r_{i}\left(\theta_{-i}\right)$ for all $i$ and $\theta_{-i}$.

Proposition $4.7 \mathbf{r}^{\pi}$ is individually undominated.

Proof. Let $i=\pi^{-1}(n)$. For all $\theta$,

$$
V C G(\theta)-\sum_{j=1, \ldots, n} r_{j}^{\pi}\left(\theta_{-j}\right) \geq \inf _{\theta_{i}^{\prime} \in \Theta_{i}}\left\{V C G\left(\theta_{i}^{\prime}, \theta_{-i}\right)-\sum_{j \neq i} r_{j}^{\pi}\left(\theta_{-j}^{\prime}\right)\right\}-r_{i}^{\pi}\left(\theta_{-i}\right)=0 .
$$

Hence $\mathbf{r}^{\pi}$ never incurs a deficit. So, $\mathbf{r}^{\pi}$ is non-deficit.

Using Proposition 4.6, we have for all $i$ and all $\theta$,

$$
\begin{gathered}
r_{i}^{\pi}\left(\theta_{-i}\right)=\inf _{\theta_{i}^{\prime} \in \Theta_{i}}\left\{V C G\left(\theta_{i}^{\prime}, \theta_{-i}\right)-\sum_{\pi(j)>\pi(i)} r_{j}\left(\theta_{-j}^{\prime}\right)-\sum_{\pi(j)<\pi(i)} r_{j}^{\pi}\left(\theta_{-j}^{\prime}\right)\right\} \\
\geq \inf _{\theta_{i}^{\prime} \in \Theta_{i}}\left\{V C G\left(\theta_{i}^{\prime}, \theta_{-i}\right)-\sum_{j \neq i} r_{j}^{\pi}\left(\theta_{-j}^{\prime}\right)\right\} .
\end{gathered}
$$

Because $\mathbf{r}^{\pi}$ is non-deficit, the opposite inequality must also be satisfied (Equation 3) - hence we must have equality, that is, Equation 4 must hold. It follows that $\mathbf{r}^{\pi}$ is individually undominated.

It should be noted that for the above technique, during the updates, we need to keep track of the value of $r_{i}^{\pi}\left(\theta_{-i}\right)$ for all $i$ and $\theta_{-i}$. That is, due to space complexity, the above technique is more suitable for cases with few agents and few possible types. To reduce 
space complexity, when we update, we could also recompute earlier updates in a recursive fashion. By doing so, the later updates are much more difficult to compute compared to the earlier updates. Fortunately, the earlier updates tend to be more important, because there is generally more room for improvement during the earlier updates. Therefore, a reasonable approximation would be to update only for a few high-priority agents and ignore the remaining agents with low priorities.

\subsection{An Iterative Technique that Preserves Anonymity}

The previous technique will, in general, not produce an anonymous mechanism, even if the input mechanism is anonymous. This is because agents higher in the priority order tend to benefit more from the technique. Here, we will introduce another technique that preserves anonymity.

Given an anonymous mechanism $r$, let $r^{0}=r$. For all $i$ and all $\theta$, let

$$
r^{k+1}\left(\theta_{-i}\right)=\frac{n-1}{n} r^{k}\left(\theta_{-i}\right)+\frac{1}{n} \inf _{\theta_{i}^{\prime} \in \Theta_{i}}\left\{V C G\left(\theta_{i}^{\prime}, \theta_{-i}\right)-\sum_{j \neq i} r^{k}\left(\theta_{-j}^{\prime}\right)\right\} .
$$

It is easily seen by induction that all the $r^{k}$ mechanisms are anonymous. If $r^{k}$ is anonymous, then for any $\pi \in \Pi(n-1), r^{k}\left(\left(\theta_{-i}\right)^{\pi}\right)=r^{k}\left(\theta_{-i}\right)$ for all $\theta$ and all $i$. We also have that $\operatorname{VCG}\left(\theta_{i}^{\prime},\left(\theta_{-i}\right)^{\pi}\right)=V C G\left(\theta_{i}^{\prime}, \theta_{-i}\right)$ for all $\theta$, all $\theta_{i}^{\prime}$, and all $i$. Finally, let $\left(\left(\theta_{-i}\right)^{\pi}, \theta_{i}^{\prime}\right)$ be the type profile where the types in $\theta_{-i}$ are permuted according to $\pi$, and $\theta_{i}$ is replaced by $\theta_{i}^{\prime}$. We have $\sum_{j \neq i} r^{k}\left(\left(\left(\theta_{-i}\right)^{\pi}, \theta_{i}^{\prime}\right)_{-j}\right)=\sum_{j \neq i} r^{k}\left(\theta_{-j}^{\prime}\right)$ for all $\theta$, all $i$, and all $\theta_{i}^{\prime}$. The above implies that $r^{k+1}$ is also permutation independent, thus anonymous.

It should be noted that from $r^{k}$ to $r^{k+1}$, agent $i$ 's payment is changed by

$$
\begin{array}{ccc} 
& & r^{k+1}\left(\theta_{-i}\right)-r^{k}\left(\theta_{-i}\right) \\
= & \frac{n-1}{n} r^{k}\left(\theta_{-i}\right)+\frac{1}{n} \inf _{\theta_{i}^{\prime} \in \Theta_{i}}\left\{V C G\left(\theta_{i}^{\prime}, \theta_{-i}\right)-\sum_{j \neq i} r^{k}\left(\theta_{-j}^{\prime}\right)\right\}-r^{k}\left(\theta_{-i}\right) \\
= & \frac{1}{n} \inf _{\theta_{i}^{\prime} \in \Theta_{i}}\left\{V C G\left(\theta_{i}^{\prime}, \theta_{-i}\right)-\sum_{j} r^{k}\left(\theta_{-j}^{\prime}\right)\right\} \\
\frac{1}{n} S_{i}^{B C G C}\left(\theta_{-i}\right) .
\end{array}
$$

That is, essentially, $r^{k+1}$ is the resulting mechanism by applying the $B C G C$ transform on $r^{k}$.

The next propositions immediately follow from Proposition 2.3:

Proposition 4.8 If $r^{0}$ is non-deficit, then $r^{k}$ is non-deficit for all $k$.

Proposition 4.9 For all $i$ and $\theta_{-i}, r^{k}\left(\theta_{-i}\right)$ is nondecreasing in $k$.

Proposition 4.10 If $r^{k+1}=r^{k}$, then $r^{k}$ is individually undominated.

Proposition 4.11 If $r^{k}$ is not individually undominated, then $r^{k+1}$ individually dominates $r^{k}$.

Finally, the following proposition establishes convergence. 
Proposition 4.12 As $k \rightarrow \infty, r^{k}$ converges (pointwise) to an individually undominated mechanism.

Proof. By Proposition 4.9, the $r^{k}\left(\theta_{-i}\right)$ are nondecreasing in $k$, and since every $r^{k}$ is nondeficit by Proposition 4.8, they must be bounded; hence they must converge (pointwise). For any $i$ and $\theta_{-i}$, let

$$
d_{k}=\inf _{\theta_{i}^{\prime} \in \Theta_{i}}\left\{V C G\left(\theta_{i}^{\prime}, \theta_{-i}\right)-\sum_{j \neq i} r^{k}\left(\theta_{-j}^{\prime}\right)\right\}-r^{k}\left(\theta_{-i}\right) .
$$

Using Proposition 4.9, we derive the following inequality:

$$
\begin{gathered}
d_{k+1}=\inf _{\theta_{i}^{\prime} \in \Theta_{i}}\left\{V C G\left(\theta_{i}^{\prime}, \theta_{-i}\right)-\sum_{j \neq i} r^{k+1}\left(\theta_{-j}^{\prime}\right)\right\}-r^{k+1}\left(\theta_{-i}\right) \\
\leq \inf _{\theta_{i}^{\prime} \in \Theta_{i}}\left\{V C G\left(\theta_{i}^{\prime}, \theta_{-i}\right)-\sum_{j \neq i} r^{k}\left(\theta_{-j}^{\prime}\right)\right\}-r^{k+1}\left(\theta_{-i}\right) \\
=\inf _{\theta_{i}^{\prime} \in \Theta_{i}}\left\{V C G\left(\theta_{i}^{\prime}, \theta_{-i}\right)-\sum_{j \neq i} r^{k}\left(\theta_{-j}^{\prime}\right)\right\} \\
=-\frac{n-1}{n} r^{k}\left(\theta_{-i}\right)-\frac{1}{n} \inf _{\theta_{i}^{\prime} \in \Theta_{i}}\left\{V C G\left(\theta_{i}^{\prime}, \theta_{-i}\right)-\sum_{j \neq i} r^{k}\left(\theta_{-j}^{\prime}\right)\right\} \\
=\frac{n-1}{n} \inf _{\theta_{i}^{\prime} \in \Theta_{i}}\left\{V C G\left(\theta_{i}^{\prime}, \theta_{-i}\right)-\sum_{j \neq i} r^{k}\left(\theta_{-j}^{\prime}\right)\right\}-\frac{n-1}{n} r^{k}\left(\theta_{-i}\right)=\frac{n-1}{n} d_{k} .
\end{gathered}
$$

As $k \rightarrow \infty, d_{k}=\inf _{\theta_{i}^{\prime} \in \Theta_{i}}\left\{V C G\left(\theta_{i}^{\prime}, \theta_{-i}\right)-\sum_{j \neq i} r^{k}\left(\theta_{-j}^{\prime}\right)\right\}-r^{k}\left(\theta_{-i}\right) \rightarrow 0$. So in the limit, Equation 4 is satisfied. Thus, $r^{k}$ converges (pointwise, linearly) to an individually undominated mechanism.

Similar to the priority-based technique, in the above iterative process, when computing for $r^{k}$, we need the value of $r^{k-1}\left(\theta_{-i}\right)$, for all $\theta_{-i}$. That is, due to space complexity, the above technique is more suitable for cases with few agents and few possible types. To reduce the space complexity we could also recompute $r^{k-1}$ in a recursive fashion. By doing so, $r^{k}$ becomes much more difficult to compute for large values of $k$. Fortunately, the earlier iterative steps are more crucial, because there is generally more room for improvement during the earlier steps. Therefore a reasonable approximation would be to only compute a few iterative steps.

\section{Multi-Unit Auctions with Unit Demand}

In this section, we consider auctions where there are multiple identical units of a single good and all agents have unit demand, i.e., each agent wants only one unit (if there is a single unit of the good, we simply have the standard single-item auction). We focus on the notion of collectively undominated mechanisms and how it relates to that of individually undominated mechanisms. In particular, we first obtain an analytical characterization of 
all collectively undominated Groves mechanisms that are non-deficit, anonymous, and have linear payment functions, by proving that the Optimal-in-Expectation Linear redistribution mechanisms (OEL) (Guo \& Conitzer, 2010), which include the BC mechanism, are the only collectively undominated Groves mechanisms that are anonymous and linear. We then show that individual undominance and collective undominance are equivalent if we restrict our consideration to Groves mechanisms that are anonymous and linear in the setting of multiunit auctions with unit demand. Note that even for single-item auctions, the examples given in Section 4.2 show that this equivalence does not hold if we do not restrict ourselves to linear and anonymous mechanisms.

If one mechanism collectively dominates another mechanism, then under the first mechanism, the agents' expected total utility, if there was a prior distribution over the agents' valuations, must be no less than that under the second mechanism, and strictly higher under minimal conditions on the prior distribution. Therefore, a good direction in which to look for collectively undominated mechanisms is to start with those mechanisms that are optimal-in-expectation.

The Optimal-in-Expectation Linear (OEL) redistribution mechanisms (Guo \& Conitzer, 2010), described below, are special cases of non-deficit Groves mechanisms that are anonymous and linear. The OEL mechanisms are defined only for multi-unit auctions with unit demand. In a unit demand multi-unit auction, there are $m$ indistinguishable units for sale, and each agent is interested in only one unit. For agent $i$, his type $\theta_{i}$ is his valuation for winning one unit. We assume all bids (announced types) are bounded below by $L$ and above by $U$, i.e., $\Theta_{i}=[L, U]$ (note that $L$ can be 0$)$.

A linear and anonymous Groves mechanism is characterized by a function $r$ of the following form: $r\left(\theta_{-i}\right)=c_{0}+\sum_{j=1}^{n-1} c_{j}\left[\theta_{-i}\right]_{j}$ (where $\left[\theta_{-i}\right]_{j}$ is the $j$ th highest bid among $\theta_{-i}$ ). For OEL mechanisms, the $c_{j}$ 's are chosen according to one of the following options (indexed by integer parameter $k$, where $k$ ranges from 0 to $n$, and $k-m$ is odd):

$\mathbf{k}=\mathbf{0}$ :

$$
\begin{gathered}
c_{i}=(-1)^{m-i}\left(\begin{array}{c}
n-i-1 \\
n-m-1
\end{array}\right) /\left(\begin{array}{c}
m-1 \\
i-1
\end{array}\right) \text { for } i=1, \ldots, m, \\
c_{0}=U m / n-U \sum_{i=1}^{m}(-1)^{m-i}\left(\begin{array}{c}
n-i-1 \\
n-m-1
\end{array}\right) /\left(\begin{array}{c}
m-1 \\
i-1
\end{array}\right), \\
c_{i}=0 \text { for other values of } i .
\end{gathered}
$$

$\mathrm{k}=1,2, \ldots, \mathrm{m}:$

$$
\begin{gathered}
c_{i}=(-1)^{m-i}\left(\begin{array}{c}
n-i-1 \\
n-m-1
\end{array}\right) /\left(\begin{array}{c}
m-1 \\
i-1
\end{array}\right) \text { for } i=k+1, \ldots, m, \\
c_{k}=m / n-\sum_{i=k+1}^{m}(-1)^{m-i}\left(\begin{array}{c}
n-i-1 \\
n-m-1
\end{array}\right) /\left(\begin{array}{c}
m-1 \\
i-1
\end{array}\right), \\
c_{i}=0 \text { for other values of } i .
\end{gathered}
$$


$\mathrm{k}=\mathbf{m}+\mathbf{1}, \mathbf{m}+\mathbf{2}, \ldots, \mathbf{n}-\mathbf{1}:$

$$
\begin{gathered}
c_{i}=(-1)^{m-i-1}\left(\begin{array}{c}
i-1 \\
m-1
\end{array}\right) /\left(\begin{array}{c}
n-m-1 \\
n-i-1
\end{array}\right) \text { for } i=m+1, \ldots, k-1, \\
c_{k}=m / n-\sum_{i=m+1}^{k-1}(-1)^{m-i-1}\left(\begin{array}{c}
i-1 \\
m-1
\end{array}\right) /\left(\begin{array}{c}
n-m-1 \\
n-i-1
\end{array}\right), \\
c_{i}=0 \text { for other values of } i .
\end{gathered}
$$

$\mathbf{k}=\mathbf{n}:$

$$
\begin{gathered}
c_{i}=(-1)^{m-i-1}\left(\begin{array}{c}
i-1 \\
m-1
\end{array}\right) /\left(\begin{array}{c}
n-m-1 \\
n-i-1
\end{array}\right) \text { for } i=m+1, \ldots, n-1, \\
c_{0}=L m / n-L \sum_{i=m+1}^{n-1}(-1)^{m-i-1}\left(\begin{array}{c}
i-1 \\
m-1
\end{array}\right) /\left(\begin{array}{c}
n-m-1 \\
n-i-1
\end{array}\right), \\
c_{i}=0 \text { for other values of } i .
\end{gathered}
$$

For example, when $k=m+1$, we have $c_{m+1}=m / n$ and $c_{i}=0$ for all other $i$. For this specific OEL mechanism, $r\left(\theta_{-i}\right)=\frac{m}{n}\left[\theta_{-i}\right]_{m+1}$. That is, besides participating in the VCG mechanism, every agent also receives an amount that is equal to $m / n$ times the $(m+1)$ th highest bid from the other agents. Actually, this is exactly the $\mathrm{BC}$ mechanism for multi-unit auctions with unit demand.

Besides being non-deficit, one property of the OEL mechanisms is that they are always budget balanced in the following scenarios.

- $[\theta]_{1}=U$ and $k=0$

- $[\theta]_{k+1}=[\theta]_{k}$ and $k \in\{1, \ldots, n-1\}$

- $[\theta]_{n}=L$ and $k=n$

Using this property, we will prove that the OEL mechanisms are the only collectively undominated non-deficit Groves mechanisms that are anonymous and linear.

We first show that the OEL mechanisms are collectively undominated.

Theorem 5.1 For multi-unit auctions with unit demand, there is no non-deficit Groves mechanism that collectively dominates an OEL mechanism.

By using Lemma 3.3, we only need to prove this for the case of anonymous Groves mechanisms.

Lemma 5.2 For multi-unit auctions with unit demand, there is no non-deficit anonymous Groves mechanism that collectively dominates an OEL mechanism. 
Proof. We first prove: no OEL mechanism with index $k \in\{1, \ldots, n-1\}$ is collectively dominated by a non-deficit anonymous Groves mechanism.

Suppose a non-deficit anonymous Groves mechanism $r$ collectively dominates an OEL mechanism with index $k \in\{1, \ldots, n-1\}$. We use $r^{O E L}$ to denote this OEL mechanism. For any $i$ and $\theta_{-i}$, we define the following function:

$$
\Delta\left(\theta_{-i}\right)=r\left(\theta_{-i}\right)-r^{O E L}\left(\theta_{-i}\right)
$$

Since $r$ collectively dominates $r O E L$, we have that for any $\theta, \sum_{i=1}^{n} \Delta\left(\theta_{-i}\right) \geq 0$.

We also have that, whenever $[\theta]_{k+1}=[\theta]_{k}$, the OEL mechanism is budget balanced. That is, under $r^{O E L}$, the agents' total payment is 0 ; in this case, since $r$ is non-deficit, we must have $\sum_{i=1}^{n} \Delta\left(\theta_{-i}\right)=0$.

Now we claim that $\Delta\left(\theta_{-i}\right)=0$ for all $\theta_{-i}$. Let $C\left(\theta_{-i}\right)$ be the number of bids among $\theta_{-i}$ that equal $\left[\theta_{-i}\right]_{k}$. Hence, we must show that for all $\theta_{-i}$ with $C\left(\theta_{-i}\right) \geq 1$, we have $\Delta\left(\theta_{-i}\right)=0$.

We now prove it by induction on the value of $C\left(\theta_{-i}\right)$ (backwards, from $n-1$ to 1 ).

Base case: $C\left(\theta_{-i}\right)=n-1$.

Suppose there is a $\theta_{-i}$ with $C\left(\theta_{-i}\right)=n-1$. That is, all the bids in $\theta_{-i}$ are identical. When $\theta_{i}$ is also equal to the bids in $\theta_{-i}$, all bids in $\theta$ are the same so that $[\theta]_{k+1}=[\theta]_{k}$. Hence, by our earlier observation, we have $\sum_{j=1}^{n} \Delta\left(\theta_{-j}\right)=0$. But we know that for all $j$, $\theta_{-j}$ is the same set of bids. Hence $\Delta\left(\theta_{-i}\right)=0$ for all $\theta_{-i}$ when $C\left(\theta_{-i}\right)=n-1$.

Induction step.

Let us assume that for all $\theta_{-i}$, if $C\left(\theta_{-i}\right) \geq p$ (where $p \in\{2, \ldots, n-1\}$ ), then $\Delta\left(\theta_{-i}\right)=0$. Now we consider any $\theta_{-i}$ with $C\left(\theta_{-i}\right)=p-1$. When $\theta_{i}$ is equal to $\left[\theta_{-i}\right]_{k}$, we have $[\theta]_{k}=$ $[\theta]_{k+1}$, which implies that $\sum_{j=1}^{n} \Delta\left(\theta_{-j}\right)=0$. For all $j$ with $\theta_{j}=\left[\theta_{-i}\right]_{k}, \Delta\left(\theta_{-j}\right)=\Delta\left(\theta_{-i}\right)$, and for other $j, C\left(\theta_{-j}\right)=p$. Therefore, by the induction assumption, $\sum_{j=1}^{n} \Delta\left(\theta_{-j}\right)$ is a positive multiple of $\Delta\left(\theta_{-i}\right)$, which implies that $\Delta\left(\theta_{-i}\right)=0$.

By induction, we have shown that $\Delta\left(\theta_{-i}\right)=0$ for all $\theta_{-i}$. This implies that $r$ and $r^{O E L}$ are identical. Hence, no other non-deficit anonymous Groves mechanism collectively dominates an OEL mechanism with index $k \in\{1, \ldots, n-1\}$.

Now we prove: the OEL mechanism with index $k=0$ is not collectively dominated by a different non-deficit anonymous Groves mechanism.

Suppose a non-deficit anonymous Groves mechanism $r$ collectively dominates an OEL mechanism with index $k=0$. We use $r^{O E L}$ to denote this OEL mechanism. For any $i$ and $\theta_{-i}$, we define the following function:

$$
\Delta\left(\theta_{-i}\right)=r\left(\theta_{-i}\right)-r^{O E L}\left(\theta_{-i}\right)
$$

Since $r$ collectively dominates $r^{O E L}$, we have that for any $\theta, \sum_{i=1}^{n} \Delta\left(\theta_{-i}\right) \geq 0$. We also have that, whenever $[\theta]_{1}=U$, under $r^{O E L}$, the agents' total payment is 0 ; in this case, because $r$ is non-deficit, we must have $\sum_{i=1}^{n} \Delta\left(\theta_{-i}\right)=0$.

Now we claim that $\Delta\left(\theta_{-i}\right)=0$ for all $\theta_{-i}$. Let $C\left(\theta_{-i}\right)$ be the number of bids among $\theta_{-i}$ that equal $U$. Hence, we must show that for all $\theta_{-i}$ with $C\left(\theta_{-i}\right) \geq 0$, we have $\Delta\left(\theta_{-i}\right)=0$.

We now prove it by induction on the value of $C\left(\theta_{-i}\right)$ (backwards, from $n-1$ to 0 ). 
Base case: $C\left(\theta_{-i}=n-1\right.$.

Suppose there is a $\theta_{-i}$ with $C\left(\theta_{-i}\right)=n-1$. That is, all the bids in $\theta_{-i}$ are equal to $U$. When $\theta_{i}$ is also equal to the bids in $U$, by our earlier observation, we have $\sum_{j=1}^{n} \Delta\left(\theta_{-j}\right)=0$. But we know that for all $j, \Delta\left(\theta_{-j}\right)$ is the same value. Hence $\Delta\left(\theta_{-i}\right)=0$ for all $\theta_{-i}$ when $C\left(\theta_{-i}\right)=n-1$.

Induction step.

Let us assume that for all $\theta_{-i}$, if $C\left(\theta_{-i}\right) \geq p$ (where $p \in\{2, \ldots, n-1\}$ ), then $\Delta\left(\theta_{-i}\right)=0$. Now we consider any $\theta_{-i}$ with $C\left(\theta_{-i}\right)=p-1$. When $\theta_{i}$ is equal to $U$, we have $[\theta]_{1}=U$, which implies that $\sum_{j=1}^{n} \Delta\left(\theta_{-j}\right)=0$. For all $j$ with $\theta_{j}=U, \Delta\left(\theta_{-j}\right)=\Delta\left(\theta_{-i}\right)$, and for other $j, C\left(\theta_{-j}\right)=p$. Therefore, by the induction assumption, $\sum_{j=1}^{n} \Delta\left(\theta_{-j}\right)$ is a positive multiple of $\Delta\left(\theta_{-i}\right)$, which implies that $\Delta\left(\theta_{-i}\right)=0$.

By induction, we have shown that $\Delta\left(\theta_{-i}\right)=0$ for all $\theta_{-i}$. This implies that $r$ and $r^{O E L}$ are identical. Hence, no other non-deficit anonymous Groves mechanism collectively dominates the OEL mechanism with index $k=0$.

It remains to prove: the OEL mechanism with index $k=n$ is not collectively dominated by a different non-deficit anonymous Groves mechanism.

This case is similar to the case of $k=0$ and we omit it here.

We now proceed to show that within the family of anonymous and linear non-deficit Groves mechanisms, the OEL mechanisms are the only ones that are collectively undominated. Actually, they are also the only ones that are individually undominated, which is a stronger claim since being individually undominated is a weaker property.

Theorem 5.3 For multi-unit auctions with unit demand, if an anonymous linear nondeficit Groves mechanism is individually undominated, then it must be an OEL mechanism.

Before proving this theorem, let us introduce the following lemma.

Lemma 5.4 Let $I$ be the set of points $\left(s_{1}, s_{2}, \ldots, s_{k}\right)\left(U \geq s_{1} \geq s_{2} \geq \ldots \geq s_{k} \geq L\right)$ that satisfy $Q_{0}+Q_{1} s_{1}+Q_{2} s_{2}+\ldots+Q_{k} s_{k}=0$ (the $Q_{i}$ are constants). If the measure of $I$ is positive (Lebesgue measure on $R^{k}$ ), then $Q_{i}=0$ for all $i$.

Proof. If $Q_{i} \neq 0$ for some $i$, then for any $U \geq s_{1} \geq s_{2} \geq \ldots \geq s_{i-1} \geq s_{i+1} \geq \ldots \geq s_{k} \geq L$, to make $Q_{0}+Q_{1} s_{1}+Q_{2} s_{2}+\ldots+Q_{k} s_{k}=0, s_{i}$ can take at most one value. As a result the measure of $I$ must be 0 .

Now we are ready to prove Theorem 5.3.

Proof. Let $r$ be a non-deficit anonymous linear Groves mechanism. We recall that a Groves mechanism is anonymous and linear if $r$ is a linear function defined as $r\left(\theta_{-i}\right)=$ $a_{0}+\sum_{j=1}^{n-1} a_{j}\left[\theta_{-i}\right]_{j}$ (where $\left[\theta_{-i}\right]_{j}$ is the $j$ th highest type among $\theta_{-i}$, and the $a_{j}$ 's are constants).

Under multi-unit auctions with unit demand, the total VCG payment equals $m[\theta]_{m+1}$ ( $m$ times the $(m+1)$ th bid). Under $r$, the agents' total payment equals

$$
m[\theta]_{m+1}-\sum_{i=1}^{n} r\left(\theta_{-i}\right)=m[\theta]_{m+1}-n a_{0}-\sum_{i=1}^{n} \sum_{j=1}^{n-1} a_{j}\left[\theta_{-i}\right]_{j} .
$$


The above total payment is a linear function in terms of the types among $\theta$. For simplicity, we rewrite the total payment as $C_{0}+C_{1}[\theta]_{1}+C_{2}[\theta]_{2}+\ldots+C_{n}[\theta]_{n}$. The $C_{i}$ are constants determined by the $a_{i}$. We have

$$
\begin{aligned}
C_{0} & =-n a_{0} \\
C_{1} & =-(n-1) a_{1} \\
C_{2} & =-a_{1}-(n-2) a_{2} \\
C_{3} & =-2 a_{2}-(n-3) a_{3} \\
& \vdots \\
C_{m} & =-(m-1) a_{m-1}-(n-m) a_{m} \\
C_{m+1} & =-m a_{m}-(n-m-1) a_{m+1}+m \\
C_{m+2} & =-(m+1) a_{m+1}-(n-m-2) a_{m+2} \\
& \vdots \\
C_{n-1} & =-(n-2) a_{n-2}-a_{n-1} \\
C_{n} & =-(n-1) a_{n-1}
\end{aligned}
$$

Given any $\theta_{-i}$, for any possible value of $\theta_{i}$, we must have $\sum_{i=1}^{n} t_{i}(\theta) \geq 0$ (non-deficit). That is, for any $\theta_{-i}$, we have $\inf _{\theta_{i}} \sum_{i=1}^{n} t_{i}(\theta) \geq 0$. If for some $\theta_{-i}$, we have $\inf _{\theta_{i}} \sum_{i=1}^{n} t_{i}(\theta)>\epsilon$ $(\epsilon>0)$, then we can reduce the payment of agent $i$ by $\epsilon$ without violating the non-deficit constraint, when the other agents' types are $\theta_{-i}$. Therefore, if the mechanism is individually undominated, then for any $\theta_{-i}$, we have $\inf _{\theta_{i}} \sum_{i=1}^{n} t_{i}(\theta)=0$.

We denote $\left[\theta_{-i}\right]_{j}$ by $s_{j}(j=1, \ldots, n-1)$. That is, $s_{1} \geq s_{2} \geq \ldots \geq s_{n-1}$.

The expression $\inf _{\theta_{i}} \sum_{i=1}^{n} t_{i}(\theta)$ then equals the minimum of the following expressions:

$$
\begin{gathered}
\inf _{L \leq \theta_{i} \leq s_{n-1}} \sum_{i=1}^{n} t_{i}(\theta) \\
\inf _{s_{n-1} \leq \theta_{i} \leq s_{n-2}} \sum_{i=1}^{n} t_{i}(\theta) \\
\vdots \\
\inf _{s_{2} \leq \theta_{i} \leq s_{1}} \sum_{i=1}^{n} t_{i}(\theta) \\
\inf _{s_{1} \leq \theta_{i} \leq U} \sum_{i=1}^{n} t_{i}(\theta)
\end{gathered}
$$


We take a closer look at $\inf _{L \leq \theta_{i} \leq s_{n-1}} \sum_{i=1}^{n} t_{i}(\theta)$. When $L \leq \theta_{i} \leq s_{n-1}$, the $j$ th highest type $[\theta]_{j}=s_{j}$ for $j=1, \ldots, n-1$, and the $n$th highest type $[\theta]_{n}=\theta_{i}$ (this case corresponds to agent $i$ being the agent with the lowest type). We have

$$
\begin{gathered}
\inf _{L \leq \theta_{i} \leq s_{n-1}} \sum_{i=1}^{n} t_{i}(\theta)=\inf _{L \leq \theta_{i} \leq s_{n-1}}\left(C_{0}+C_{1} s_{1}+C_{2} s_{2}+\ldots+C_{n-1} s_{n-1}+C_{n} \theta_{i}\right) \\
=\min \left\{C_{0}+C_{1} s_{1}+\ldots+C_{n-1} s_{n-1}+C_{n} L, C_{0}+C_{1} s_{1}+\ldots+C_{n-1} s_{n-1}+C_{n} s_{n-1}\right\} .
\end{gathered}
$$

That is, because the expression is linear, the minimum is reached when $\theta_{i}$ is set to either the lower bound $L$ or the upper bound $s_{n-1}$.

Similarly, we have

$$
\begin{gathered}
\inf _{s_{n-1} \leq \theta_{i} \leq s_{n-2}} \sum_{i=1}^{n} t_{i}(\theta)=\min \left\{C_{0}+C_{1} s_{1}+\ldots+C_{n-2} s_{n-2}+C_{n-1} s_{n-1}+C_{n} s_{n-1},\right. \\
\left.C_{0}+C_{1} s_{1}+C_{2} s_{2}+\ldots+C_{n-2} s_{n-2}+C_{n-1} s_{n-2}+C_{n} s_{n-1}\right\} \\
\vdots \\
\inf _{s_{2} \leq \theta_{i} \leq s_{1}} \sum_{i=1}^{n} t_{i}(\theta)=\min \left\{C_{0}+C_{1} s_{1}+C_{2} s_{1}+C_{3} s_{2}+\ldots+C_{n} s_{n-1},\right. \\
\left.C_{0}+C_{1} s_{1}+C_{2} s_{2}+C_{3} s_{2}+\ldots+C_{n} s_{n-1}\right\} \\
\inf _{s_{1} \leq \theta_{i} \leq U} \sum_{i=1}^{n} t_{i}(\theta)=\min \left\{C_{0}+C_{1} U+C_{2} s_{1}+\ldots+C_{n} s_{n-1}\right. \\
\left.C_{0}+C_{1} s_{1}+C_{2} s_{1}+\ldots+C_{n} s_{n-1}\right\} .
\end{gathered}
$$

Putting all the above together, we have that for any $U \geq s_{1} \geq s_{2} \geq \ldots \geq s_{n-1} \geq L$, the minimum of the following expressions is 0 .

- $(n): C_{0}+C_{1} s_{1}+C_{2} s_{2}+\ldots+C_{n-1} s_{n-1}+C_{n} L$

- $(n-1): C_{0}+C_{1} s_{1}+C_{2} s_{2}+\ldots+C_{n-1} s_{n-1}+C_{n} s_{n-1}$

- $(n-2): C_{0}+C_{1} s_{1}+C_{2} s_{2}+\ldots+C_{n-2} s_{n-2}+C_{n-1} s_{n-2}+C_{n} s_{n-1}$

- :

- (2): $C_{0}+C_{1} s_{1}+C_{2} s_{2}+C_{3} s_{2}+\ldots+C_{n} s_{n-1}$

- (1): $C_{0}+C_{1} s_{1}+C_{2} s_{1}+C_{3} s_{2}+\ldots+C_{n} s_{n-1}$

- (0): $C_{0}+C_{1} U+C_{2} s_{1}+C_{3} s_{2}+\ldots+C_{n} s_{n-1}$ 
The above expressions are numbered from 0 to $n$. Let $I(i)$ be the set of points $\left(s_{1}, \ldots, s_{n-1}\right)$ $\left(U \geq s_{1} \geq s_{2} \geq \ldots \geq s_{n-1} \geq L\right)$ that make expression $(i)$ equal to 0 . There must exist at least one $i$ such that the measure of $I(i)$ is positive. According to Lemma 5.4, expression (i) must be the constant 0 .

If expression ( 0 ) is constant 0 , then the total payment under $r$ is 0 whenever the highest type is equal to the upper bound $U$. That is, for any $\theta$, the total payment $C_{0}+C_{1}[\theta]_{1}+$ $C_{2}[\theta]_{2}+\ldots+C_{n}[\theta]_{n}$ must be a constant multiple of $U-[\theta]_{1}$ (the total payment is a linear function). We have $C_{0}=-U C_{1}$ and $C_{j}=0$ for $j \geq 2$. It turns out that the above equalities of the $C_{j}$ completely determine the values of the $a_{j}$ (the values of the $a_{j}$ can be solved for based on the $C_{j}$ by pure algebraic manipulations), and the corresponding mechanism is the OEL mechanism with index $k=0$. If expression $(i)$ is constant for other values of $i$, then the corresponding mechanism is the OEL mechanism with another index.

Hence, we have the following complete characterization in this context.

Corollary 5.5 For multi-unit auctions with unit demand, a non-deficit anonymous linear Groves mechanism is individually / collectively undominated if and only if it is an OEL mechanism.

Proof. This corollary can be proved by combining Theorem 5.1 and Theorem 5.3, as well as the fact that a collectively undominated mechanism is also individually undominated.

The above corollary also shows that if we consider only Groves mechanisms that are non-deficit, anonymous, and linear in the setting of multi-unit auctions with unit demand, then individual undominance and collective undominance are equivalent. Thus, we have characterized all individually/collectively undominated Groves mechanisms that are nondeficit, anonymous, and linear for multi-unit auctions with unit demand.

\section{The Public Project Problem}

We now study a well known class of decision problems, namely public project problems (see, e.g., Mas-Colell et al., 1995; Moulin, 1988; Moore, 2006). In this setting a set of $n$ agents needs to decide on financing a project of $\operatorname{cost} c$. An agent's type is her private valuation for the project if it takes place. We consider two versions of the problem.

\subsection{Equal Participation Costs}

In this case if the project takes place, each agent contributes the same share, $c / n$, so as to cover the total cost. Hence the participation costs of all agents are the same. So the problem is defined as follows.

Public project problem

Consider $\left(D, \Theta_{1}, \ldots, \Theta_{n}, v_{1}, \ldots, v_{n}\right)$, where

- $D=\{0,1\}$ (reflecting whether a project is canceled or takes place),

- for all $i \in\{1, \ldots, n\}, \Theta_{i}=[0, c]$, where $c>0$, 
- for all $i \in\{1, \ldots, n\}, v_{i}\left(d, \theta_{i}\right):=d\left(\theta_{i}-\frac{c}{n}\right)$,

When the agents employ a payment-based mechanism to decide on the project, then in addition to $c / n$, each agent also has to pay or receive the payment, $t_{i}(\theta)$, imposed by the mechanism. By the result of Holmström (1979), the only efficient and strategyproof payment-based mechanisms in this domain are Groves mechanisms. To determine the efficient outcome for a given type vector $\theta$, note that $\sum_{i=1}^{n} v_{i}\left(d, \theta_{i}\right)=d\left(\sum_{i=1}^{n} \theta_{i}-c\right)$. Hence efficiency here for a mechanism $(f, t)$ means that $f(\theta)=1$ if $\sum_{i=1}^{n} \theta_{i} \geq c$ and $f(\theta)=0$ otherwise, i.e., the project takes place if and only if the declared total value that the agents have for the project exceeds its cost.

We first observe the following result.

Proposition 6.1 In the public project problem with equal participation costs, the BC mechanism coincides with VCG.

Proof. It suffices to check that in equation (1) it holds that $S_{i}^{B C G C}\left(\theta_{-i}\right)=0$ for all $i$ and all $\theta_{-i}$. Since VCG is a non-deficit mechanism, we have $S_{i}^{B C G C}\left(\theta_{-i}\right) \geq 0$, as the term $S_{i}^{B C G C}\left(\theta_{-i}\right)$ is a sum of payments for some type vector. Hence all we need is to show that there is a value for $\theta_{i}^{\prime}$ that makes the expression in (1) equal to 0. Checking this is quite simple. If $\sum_{j \neq i} \theta_{j}<\frac{n-1}{n} c$, then we take $\theta_{i}^{\prime}:=0$ and otherwise $\theta_{i}^{\prime}:=c$. In the former case the efficient outcome is to not implement the project whereas in the latter case, the opposite occurs. It is easy to check that in both cases we have $S_{i}^{B C G C}\left(\theta_{-i}\right)=0$.

We now show that in fact VCG cannot be improved upon. Before stating our result, we would like to note that one ideally would like to have a mechanism that is budget-balanced, i.e., $\sum_{i} t_{i}(\theta)=0$ for all $\theta$, so that in total the agents only pay the cost of the project and no more. However this is not possible, since for the public project problem, no mechanism exists that is efficient, strategy-proof, and budget balanced (Mas-Colell et al., 1995). Our theorem below considerably strengthens this result, showing that VCG is optimal with respect to minimizing the total payment of the agents.

Theorem 6.2 In the public project problem there exists no non-deficit Groves mechanism that collectively dominates the VCG mechanism.

As with the case of unit-demand auctions, we first establish the desired conclusion for anonymous Groves mechanisms and then extend it to arbitrary ones by Lemma 3.3. Notice that VCG is anonymous in this setting and hence we can apply Lemma 3.3(ii).

Lemma 6.3 In the public project problem there exists no anonymous non-deficit Groves mechanism that collectively dominates the VCG mechanism.

Proof. Suppose that an anonymous non-deficit Groves mechanism $\left(r_{1}, \ldots, r_{n}\right)$ exists that collectively dominates VCG. By anonymity, for all $i \in\{1, \ldots, n\} \quad r_{i}=r$, for some function $r:[0, c]^{n-1} \rightarrow \mathbb{R}$. Hence

$$
\forall \theta \in[0, c]^{n} \quad \sum_{i=1}^{n} r\left(\theta_{-i}\right) \geq 0
$$


We will show that then for all $x \in[0, c]^{n-1}, r(x)=0$ and thus $r$ coincides with VCG.

We divide our proof into two cases.

Case 1: The vector $x$ satisfies $\sum_{i=1}^{n-1} x_{i} \geq \frac{n-1}{n} c$.

Given such an $x$, define $C(x)=\left|\left\{i: x_{i}=c\right\}\right|$, i.e., given a vector $x$ of $n-1$ types, $C(x)$ is the number of agents who submitted $c$. Define the following predicate:

$$
P(k): \forall x \in[0, c]^{n-1}\left(\left(C(x)=k \wedge \sum_{i=1}^{n-1} x_{i} \geq \frac{n-1}{n} c\right) \rightarrow r(x)=0\right)
$$

We now prove that $P(k)$ holds for all $k \in\{0, \ldots, n-1\}$, using induction (going backwards from $n-1)$. Let $t_{i}(\theta)=V C G_{i}(\theta)-r\left(\theta_{-i}\right)$ be the payment function of agent $i$ under the mechanism $r$.

\section{Base case.}

Let $x$ be such that $C(x)=n-1$. Consider $\theta:=(c, \ldots, c) \in[0, c]^{n}$. Then for all $i \in\{1, \ldots, n\}, \theta_{-i}=x$. Clearly $f(\theta)=1$ and no agent is paying anything under the VCG mechanism in this instance, i.e., $V C G_{i}(\theta)=0$.

Since $r$ is a non-deficit mechanism

$$
0 \leq \sum_{i=1}^{n} t_{i}(\theta)=\sum_{i=1}^{n} V C G_{i}(\theta)-\sum_{i=1}^{n} r\left(\theta_{-i}\right)=-\sum_{i=1}^{n} r\left(\theta_{-i}\right)=-n r(x),
$$

But then by (6) we have $r(x)=0$.

\section{Induction step.}

Assume $P(k)$ holds for some $k \geq 1$. We will prove $P(k-1)$. Let $x$ be such that $C(x)=k-1$ (note that $x$ may have zero $c$ 's). Since $r$ is permutation independent, we can assume without loss of generality that the elements of $x$ are sorted in descending order (i.e., $r(x)$ does not change by such a reordering). Consider the type vector $\theta=(c, x)$, that is the concatenation of $(c)$ and $x$. Hence $\theta$ starts with $k c$ 's and the rest is like the rest of $x$. Note that for $i \in\{1, \ldots, k\}, \theta_{-i}=x$ and $C\left(\theta_{-i}\right)=k-1$. For $i \in\{k+1, \ldots, n\}, C\left(\theta_{-i}\right)=k$, therefore by induction hypothesis, $r\left(\theta_{-i}\right)=0$. This means that $\sum_{i=1}^{n} r\left(\theta_{-i}\right)=k r(x)$.

Furthermore, $f(\theta)=1$ since $\theta$ has at least one $c$, and no agent is paying payment under the VCG mechanism. To see this, if $k \geq 2$, then for every agent under $\theta$, there is another agent who submitted $c$ hence the agent is not pivotal. If $k=1$, then no agent can alter the decision outcome by the fact that $\sum x_{i} \geq \frac{n-1}{n} \cdot c$, hence no agent is pivotal in this case as well. Thus, for all $i \in\{1, \ldots, n\}, V C G_{i}(\theta)=0$, and because $r$ is non-deficit

$$
0 \leq \sum_{i=1}^{n} t_{i}(\theta)=-\sum_{i=1}^{n} r\left(\theta_{-i}\right)=-k r(x)
$$

But then by (6) we have that $r(x)=0$. This concludes the induction step and consequently $r(x)=0$ for all vectors $x$ that belong to Case 1 .

Case 2: The vector $x$ satisfies $\sum_{i=0}^{n-1} x_{i}<\frac{n-1}{n} c$. The proof for this case uses a completely symmetric argument to that of Case 1 . We include it below for the sake of completeness. 
Define $C^{\prime}(x)=\left|\left\{i: x_{i}=0\right\}\right|$. In analogy to the predicate $P(k)$ of Case 1 , we define the following predicate:

$$
P^{\prime}(k): \forall x \in[0, c]^{n-1}\left(\left(C^{\prime}(x)=k \wedge \sum_{i=0}^{n-1} x_{i}<\frac{n-1}{n} c\right) \rightarrow r(x)=0\right)
$$

We now prove that $P^{\prime}(k)$ holds for all $k \in\{0, \ldots, n-1\}$, using induction (going backwards from $n-1)$.

\section{Base case.}

Let $x$ be such that $C^{\prime}(x)=n-1$, i.e., the zero vector. Consider $\theta:=(0, \ldots, 0) \in[0, c]^{n}$. Then for all $i \in\{1, \ldots, n\}, \theta_{-i}=x$. Clearly $f(\theta)=0$ and no agent is paying anything under the VCG mechanism. Hence if $t_{i}(\theta)$ is the payment paid by agent $i$, then $\sum t_{i}(\theta)=$ $-\sum r\left(\theta_{-i}\right)=-n r(x)$.

Since $r$ is a non-deficit mechanism,

$$
0 \leq \sum_{i=1}^{n} t_{i}(\theta)=-n r(x)
$$

Then by (6) this implies that $r(x)=0$.

\section{Induction step.}

Suppose $P^{\prime}(k)$ holds for some $k \geq 1$. We will prove $P^{\prime}(k-1)$. Let $x$ be such that $C^{\prime}(x)=k-1$. Since $r$ is permutation independent, we can assume without loss of generality that the elements of $x$ are sorted in increasing order so that all 0 's are on the left side of $x$ (note that it may also be that $x$ does not have any 0 's, since $k-1$ maybe equal to 0 ). Consider the type vector $\theta=(0, x)$. So $\theta$ starts with $k 0$ 's and the rest is like the rest of $x$. Note that for $i \in\{1, \ldots, k\}, \theta_{-i}=x$ and $C^{\prime}\left(\theta_{-i}\right)=k-1$. For $i \in\{k+1, \ldots, n\}, C^{\prime}\left(\theta_{-i}\right)=k$ and by induction hypothesis, $r\left(\theta_{-i}\right)=0$ and hence $\sum r\left(\theta_{-i}\right)=k r(x)$.

We note that $f(\theta)=0$ and that also no agent is paying payment under the VCG mechanism. To see this, it is enough to verify that no agent is pivotal, which follows by the fact that we are in the case that $\sum_{i=0}^{n-1} x_{i}<\frac{n-1}{n} c$. Since $\theta=(0, x)$, no agent can be pivotal. Therefore $V C G_{i}(\theta)=0$ for every $i \in\{1, \ldots, n\}$. Since $r$ is non-deficit we have $0 \leq-\sum r\left(\theta_{-i}\right)=-k r(x)$. By (6) we have $r(x)=0$.

This completes the proof of the induction step and hence Case 2. Since Cases 1 and 2 cover all vectors $x \in[0, c]^{n-1}$, the proof of the Lemma is complete.

By using now Lemma 6.3 and Lemma 3.3(ii), the proof of Theorem 6.2 is complete.

An interesting open question is whether other mechanisms that share some of the properties of the VCG mechanism are also collectively undominated. In particular, we have exhibited that VCG is a pay-only and anonymous mechanism. Are there other anonymous or pay-only mechanisms that are collectively undominated for the public project problem with equal participation costs?

We start with pay-only mechanisms. We provide a general observation that holds in many domains other than public project problems, showing that the VCG mechanism dominates all other pay-only mechanisms. 
Lemma 6.4 Let $\boldsymbol{r}$ be a Groves mechanism. Suppose that the following condition ${ }^{11}$ holds for all $i \in\{1, \ldots, n\}$ :

$$
\forall \theta_{-i} \in \Theta_{-i} \exists b_{i}^{*} \in \Theta_{i} \text { such that } V C G_{i}\left(b_{i}^{*}, \theta_{-i}\right)-r_{i}\left(\theta_{-i}\right)=0 .
$$

Then $\boldsymbol{r}$ individually dominates all other pay-only Groves mechanisms.

The condition essentially says that every agent is always able to make his payment equal to 0 for any type vector $\theta_{-i}$ of the other agents.

Proof. Suppose that there exists a pay-only mechanism $\mathbf{r}^{\prime}=\left(r_{1}^{\prime}, \ldots, r_{n}^{\prime}\right)$ different from $\mathbf{r}=\left(r_{1}, \ldots, r_{n}\right)$ and not dominated by $\mathbf{r}$. Then, for some $\theta \in \Theta$ and $i \in\{1, \ldots n\}, r_{i}^{\prime}\left(\theta_{-i}\right)>$ $r_{i}\left(\theta_{-i}\right)$. Let $b_{i}^{*}$ be the type of agent $i$ that satisfies the condition of the theorem. Consider $\theta^{\prime}=\left(b_{i}^{*}, \theta_{-i}\right)$. Then $V C G_{i}\left(\theta^{\prime}\right)=r_{i}\left(\theta_{-i}\right)$.

But then the payment of agent $i$ under mechanism $\mathbf{r}^{\prime}$ for the profile $\theta^{\prime}$ is

$$
t_{i}^{\prime}\left(\theta^{\prime}\right)=V C G_{i}\left(\theta^{\prime}\right)-r_{i}^{\prime}\left(\theta_{-i}\right)<V C G_{i}\left(\theta^{\prime}\right)-r_{i}\left(\theta_{-i}\right)=0,
$$

which is a contradiction, because $\mathbf{r}^{\prime}$ is a pay-only mechanism.

Theorem 6.5 Consider the public project problem with equal participation costs. Then for a pay-only Groves mechanism $\boldsymbol{r}$, the following are equivalent:

1. $\boldsymbol{r}$ is individually undominated,

2. $\boldsymbol{r}$ is the VCG mechanism,

3. $\boldsymbol{r}$ is collectively undominated.

Proof. $1 \rightarrow 2$. Consider a pay-only and individually undominated Groves mechanism $\mathbf{r}$. We claim that $\mathbf{r}$ is the VCG mechanism.

In the considered domain every agent $i$, given $\theta_{-i}$, can force his VCG payment to be 0 by declaring $b_{i}^{*}=c / n$. Indeed, we then would have $V C G_{i}\left(c / n, \theta_{-i}\right)=0$. Hence by Lemma 6.4 the VCG mechanism individually dominates all other pay-only mechanisms. This means that there can be no other individually undominated mechanism than VCG.

$2 \rightarrow 3$ holds by Theorem 6.2 and $3 \rightarrow 1$ holds by the definition.

The above theorem shows that for the public project problem with equal participation costs, VCG is the only pay-only Groves mechanism that is individually/collectively undominated. In Appendix A, we show a similar result for anonymous Groves mechanisms, but only for the case of two agents. That is, if there are exactly two agents, VCG is the only anonymous Groves mechanism that is individually/collectively undominated. Further, for $n \geq 3$, Hervé Moulin (private communication) observed that for public project problems with equal participation costs, the VCG mechanism is not the only non-deficit Groves mechanism that is collectively undominated.

11. This is a slight generalization of the Potential for Universal Relevance Nullification (PURN) condition introduced by Cavallo (2006). An agent satisfies PURN if he can make his payment under the VCG mechanism equal to 0 for any type vector $\theta_{-i}$ of the other agents. Here, the only difference is that we consider all Groves mechanisms instead of just VCG. 


\subsection{The General Case}

The assumption that we have made so far in the public project problem that each agent's cost share is the same may not always be realistic. Indeed, it may be argued that 'richer' agents (such as larger enterprises) should contribute more. Does it matter if we modify the formulation of the problem appropriately? The answer is 'yes'. First, let us formalize this version of the problem. We assume now that each initial utility function is of the form

$$
v_{i}\left(d, \theta_{i}\right):=d\left(\theta_{i}-c_{i}\right),
$$

where for all $i \in\{1, \ldots, n\}, c_{i}>0$ and $\sum_{i=1}^{n} c_{i}=c$.

In this setting, $c_{i}$ is the share of the project cost to be financed by agent $i$. We call the resulting problem the general public project problem. It is taken from Moore (2006). For this problem we have only two results, both concerning the individual dominance relation.

Theorem 6.6 In the general public project problem the VCG mechanism individually dominates all other pay-only Groves mechanisms.

Proof. Note that for any $i$ and any $\theta_{-i}$, agent $i$ can force his VCG payment to be 0 by declaring $c_{i}$, since $t_{i}\left(c_{i}, \theta_{-i}\right)=0$. By Lemma 6.4 the proof is complete.

The above theorem cannot be extended to non-deficit Groves mechanisms, as is illustrated by the following theorem. The theorem below also shows that if there is an individually undominated mechanism in this setting, it cannot be a pay-only mechanism.

Theorem 6.7 For any $n \geq 3$, an instance of the general public project problem with $n$ agents exists for which the BC mechanism individually dominates the VCG mechanism.

Proof. We will show this for $n=3$. For $n>3$, it is fairly simple to extend the proof. We omit the details. The VCG mechanism is non-deficit, hence it suffices to show by Proposition 2.3(ii) that the VCG and BC mechanisms do not coincide, for some choice of $c, c_{1}, c_{2}, c_{3}$, with $c_{1}+c_{2}+c_{3}=c$.

To this end we need to find $\theta_{2}$ and $\theta_{3}$ so that $S_{1}^{B C G C}\left(\theta_{2}, \theta_{3}\right)>0$. Here

$$
S_{1}^{B C G C}\left(\theta_{2}, \theta_{3}\right):=\min _{\theta_{1}^{\prime} \in \Theta_{1}}\left(\left(R_{1}+R_{2}+R_{3}\right)-L\right),
$$

where for $\theta^{\prime}:=\left(\theta_{1}^{\prime}, \theta_{2}, \theta_{3}\right)$

$$
\begin{gathered}
L:=(n-1) \sum_{k=1}^{n} v_{k}\left(f\left(\theta^{\prime}\right), \theta_{k}^{\prime}\right), \\
R_{1}=\max _{d \in D} \sum_{j \neq 1} v_{j}\left(d, \theta_{j}^{\prime}\right)=\max \left\{0, \theta_{2}+\theta_{3}-\left(c_{2}+c_{3}\right)\right\}, \\
R_{2}=\max _{d \in D} \sum_{j \neq 2} v_{j}\left(d, \theta_{j}^{\prime}\right)=\max \left\{0, \theta_{1}^{\prime}+\theta_{3}-\left(c_{1}+c_{3}\right)\right\}, \\
R_{3}=\max _{d \in D} \sum_{j \neq 3} v_{j}\left(d, \theta_{j}^{\prime}\right)=\max \left\{0, \theta_{1}^{\prime}+\theta_{2}-\left(c_{1}+c_{2}\right)\right\} .
\end{gathered}
$$


Now, take $c=100, c_{1}=10, c_{2}=40, c_{3}=50$ and $\theta_{2}:=10, \theta_{3}:=70$. Then $R_{1}+R_{2}+R_{3}=$ $\theta_{1}^{\prime}+10+\max \left\{0, \theta_{1}^{\prime}-40\right\}$. Two cases arise.

Case $1 f\left(\theta^{\prime}\right)=0$.

Then $L=0$, so $\left(R_{1}+R_{2}+R_{3}\right)-L \geq 10$.

Case $2 f\left(\theta^{\prime}\right)=1$.

Then $L=2\left(\theta_{1}^{\prime}+\theta_{2}+\theta_{3}-100\right)=2 \theta_{1}^{\prime}-40$, so

$$
\begin{aligned}
\left(R_{1}+R_{2}+R_{3}\right)-L & =50-\theta_{1}^{\prime}+\max \left\{0, \theta_{1}^{\prime}-40\right\} \\
& \geq\left(50-\theta_{1}^{\prime}\right)+\left(\theta_{1}^{\prime}-40\right) \geq 10 .
\end{aligned}
$$

This proves that $S_{1}^{B C G C}\left(\theta_{2}, \theta_{3}\right) \geq 10$. By taking any $\theta_{1}^{\prime} \in[40,100]$ we see that in fact $S_{1}^{B C G C}\left(\theta_{2}, \theta_{3}\right)=10$.

By virtue of Theorem 6.6 the $\mathrm{BC}$ mechanism in the above proof is not pay-only.

\section{Conclusions and Future Work}

The family of Groves mechanisms, which includes the well-known VCG mechanism (also known as the Clarke mechanism), is a family of efficient and strategy-proof mechanisms. Unfortunately, the Groves mechanisms are generally not budget balanced. That is, under such mechanisms, payments may flow into or out of the system of the agents, resulting in deficits or reduced utilities for the agents. To identify non-deficit Groves mechanisms that give the agents the highest utilities, we introduced two general measures for comparing mechanisms in prior-free settings. Specifically, we say that a non-deficit Groves mechanism $M$ individually dominates another non-deficit Groves mechanism $M^{\prime}$ if for every type profile, every agent's utility under $M$ is no less than that under $M^{\prime}$, and this holds with strict inequality for at least one type profile and one agent. We say that a non-deficit Groves mechanism $M$ collectively dominates another non-deficit Groves mechanism $M^{\prime}$ if for every type profile, the agents' total utility (social welfare) under $M$ is no less than that under $M^{\prime}$, and this holds with strict inequality for at least one type profile. The above definitions induce two partial orders on non-deficit Groves mechanisms. This paper mainly focused on studying the maximal elements corresponding to these two partial orders.

A number of interesting open problems remain. Specifically,

- We provided in Section 4.2 two examples showing that collective undominance is strictly stronger than individual undominance. One example involves a discrete type space, while the other example involves discontinuous redistribution functions. It remains to be seen whether the two definitions of undominance coincide when the type space is smoothly connected and the redistribution functions are continuous.

- We know from Guo and Conitzer (2010) that the OEL mechanisms are not the only collectively undominated mechanisms in multi-unit auctions with unit demand, because there exist prior distributions under which other mechanisms achieve strictly higher expected social welfare. That is, for multi-unit auctions with unit demand, there exist other unknown collectively undominated mechanisms (based on nonlinear redistribution functions). However, it remains to be seen whether there also exist collectively undominated mechanisms (other than VCG) for public project problems. 
- We proposed two techniques for generating individually undominated mechanisms. Can we also derive techniques for generating collectively undominated mechanisms?

\section{Acknowledgments}

The authors would like to thank all three reviewers for their useful comments. We also thank Hervé Moulin for valuable discussions. This work has been supported by the project DIACODEM of the Dutch organization for scientific research (NWO), and by the project AGT of the research funding program THALIS (co-financed by the European Social FundESF and Greek national funds). We also thank the National Science Foundation and the Alfred P. Sloan Foundation for support under Awards IIS-0812113, IIS-0953756, and CCF1101659, and a Sloan Fellowship.

\section{Appendix A. Uniqueness of VCG for the Case of Two Agents}

Theorem A.1 Consider the public project problem with equal participation costs. When the number of agents is $n=2$, then for a non-deficit, and anonymous Groves mechanism $\boldsymbol{r}$, the following are equivalent:

1. $\boldsymbol{r}$ is individually undominated,

2. $\boldsymbol{r}$ is the VCG mechanism,

3. $\boldsymbol{r}$ is collectively undominated.

Proof. As in the proof of Theorem 6.5 it suffices to show that $1 \rightarrow 2$. So take a non-deficit, anonymous, and individually undominated Groves mechanism, determined by the function $r$.

For $x \in[0, c]$, take $\theta:=(x, x)$. If $x \geq c / 2$, then the efficient outcome is $f(\theta)=1$ and no agent is pivotal, hence the total VCG payment is 0 . If $x<c / 2$, then the project is not built and again no agent is pivotal. Hence in both cases the VCG payment is 0. If $t$ is the payment function corresponding to $r$, then we have that $t_{1}(\theta)+t_{2}(\theta)=-2 r(x)$. Since $r$ is non-deficit, we have that for every $x \in[0, c], r(x) \leq 0$. But since $r$ is individually undominated, it cannot be the case that $r(x)<0$ for some $x$, because then the VCG mechanism would dominate $r$. Hence $r$ coincides with the VCG mechanism.

\section{References}

Apt, K., Conitzer, V., Guo, M., \& Markakis, E. (2008). Welfare undominated Groves mechanisms. In Proceedings of the Fourth Workshop on Internet and Network Economics (WINE), pp. 426-437, Shanghai, China.

Bailey, M. J. (1997). The demand revealing process: to distribute the surplus. Public Choice, 91, 107-126.

Cavallo, R. (2006). Optimal decision-making with minimal waste: Strategyproof redistribution of VCG payments. In Proceedings of the International Conference on Autonomous Agents and Multi-Agent Systems (AAMAS), pp. 882-889, Hakodate, Japan. 
Clarke, E. H. (1971). Multipart pricing of public goods. Public Choice, 11, 17-33.

Cramton, P., Gibbons, R., \& Klemperer, P. (1987). Dissolving a partnership efficiently. Econometrica, 55(3), 615-632.

de Clippel, G., Naroditskiy, V., \& Greenwald, A. (2009). Destroy to save. In Proceedings of the ACM Conference on Electronic Commerce (EC), pp. 207-214, Stanford, CA, USA.

Faltings, B. (2005). A budget-balanced, incentive-compatible scheme for social choice. In Agent-Mediated Electronic Commerce (AMEC), LNAI, 3435, pp. 30-43.

Groves, T. (1973). Incentives in teams. Econometrica, 41, 617-631.

Gujar, S., \& Narahari, Y. (2011). Redistribution mechanisms for assignment of heterogeneous objects. J. Artif. Intell. Res. (JAIR), 41, 131-154.

Guo, M. (2011). VCG redistribution with gross substitutes. In Proceedings of the National Conference on Artificial Intelligence (AAAI), San Francisco, CA, USA.

Guo, M. (2012). Worst-case optimal redistribution of VCG payments in heterogeneousitem auctions with unit demand. In Proceedings of the Eleventh International Joint Conference on Autonomous Agents and Multi-Agent Systems (AAMAS), Valencia, Spain.

Guo, M., \& Conitzer, V. (2008a). Better redistribution with inefficient allocation in multiunit auctions with unit demand. In Proceedings of the ACM Conference on Electronic Commerce (EC), pp. 210-219, Chicago, IL, USA.

Guo, M., \& Conitzer, V. (2008b). Undominated VCG redistribution mechanisms. In Proceedings of the Seventh International Joint Conference on Autonomous Agents and Multi-Agent Systems (AAMAS), pp. 1039-1046, Estoril, Portugal.

Guo, M., \& Conitzer, V. (2009). Worst-case optimal redistribution of VCG payments in multi-unit auctions. Games and Economic Behavior, 67(1), 69-98.

Guo, M., \& Conitzer, V. (2010). Optimal-in-expectation redistribution mechanisms. Artificial Intelligence, $174(5-6), 363-381$.

Guo, M., Naroditskiy, V., Conitzer, V., Greenwald, A., \& Jennings, N. R. (2011). Budgetbalanced and nearly efficient randomized mechanisms: Public goods and beyond. In Proceedings of the Seventh Workshop on Internet and Network Economics (WINE), Singapore.

Holmström, B. (1979). Groves' scheme on restricted domains. Econometrica, 47(5), 11371144.

Laffont, J., \& Maskin, E. (1997). The theory of incentives: An overview, in: W. Hildenbrand, ed., Advances in economics theory, Econometric Society Monograph in Quantitative Economics. Cambridge University Press.

Mas-Colell, A., Whinston, M., \& Green, J. R. (1995). Microeconomic Theory. Oxford University Press.

Moore, J. (2006). General Equilibrium and Welfare Economics: An Introduction. Springer.

Moulin, H. (1988). Axioms of Cooperative Decision Making. Cambridge University Press. 
Moulin, H. (1986). Characterizations of the pivotal mechanism. Journal of Public Economics, 31(1), 53-78.

Moulin, H. (2009). Almost budget-balanced VCG mechanisms to assign multiple objects. Journal of Economic Theory, 144(1), 96-119.

Myerson, R., \& Satterthwaite, M. (1983). Efficient mechanisms for bilateral trading. Journal of Economic Theory, 28, 265-281.

Porter, R., Shoham, Y., \& Tennenholtz, M. (2004). Fair imposition. Journal of Economic Theory, 118, 209-228. 